\title{
The spread and control of rumors in a multilingual environment
}

\author{
Shuzhen Yu · Zhiyong Yu · Haijun Jiang® - Xuehui Mei · Jiarong Li
}

Received: 17 December 2019 / Accepted: 2 April 2020 / Published online: 27 April 2020

(C) Springer Nature B.V. 2020

\begin{abstract}
This paper studies the rumor propagation model with heterogeneous networks in a multilingual environment. Firstly, a rumor propagation model with two language spreaders, in which the immunologic mechanism is considered in the ignorant, is proposed on heterogeneous networks. Secondly, the basic reproduction number and the dynamic behaviors are analyzed by using the next-generation matrix method and Lyapunov stability theory, respectively. Moreover, two control strategies are designed to effectively suppress the spread of the rumor. The one is continuous control strategy. By applying real-time control to the spreaders, the rumor spreading time can be greatly reduced and the rumor can die out in a short time. The other is event-triggered impulsive control strategy, which can effectively reduce the consumption of resources and ensure the extinction of the rumor. Finally, the correctness of theoretical analysis and the feasibility of control methods are verified by numerical simulations.
\end{abstract}

Keywords Rumor spreading · Heterogeneous network · Multilingual environment · Continuous control $\cdot$ Event-triggered impulsive control

S. Yu $\cdot$ Z. Yu $\cdot$ H. Jiang $(\varangle) \cdot$ X. Mei $\cdot$ J. Li

College of Mathematics and System Sciences, Xinjiang University, Urumqi 830046, People's Republic of China e-mail: jianghaijunxju@163.com; jianghai@xju.edu.cn

\section{Introduction}

The rumor is unconfirmed information of public opinion, which greatly affects people's work and life. With the rapid development of Internet information technology, rumor propagation is no longer limited to word of mouth, but relies on the new media of the Internet, such as BBS, SMS, Microblog, QQ, WeChat, Twitter and other social network platforms. For the past few years, a number of emergencies caused by rumors have erupted in China, which have brought huge economic losses to the country and seriously disturbed people's life order. For example, in 2003, the rumor that banlangen could cure SARS caused residents in many cities around the country to frantically buy up banlangen, which caused a great social panic. In the 2011 nuclear disaster in Japan, the rumor that iodized salt could protect against radiation caused the run on salt. These rumors seriously destroyed the social order and caused social panic. With the advent of information dissemination, there are more and more emergencies caused by rumors. Therefore, it is very significant to study and master the law of rumor propagation in the new era, which can help the government department to prevent and control rumors.

Since the form of rumor transmission is similar to disease transmission, based on the disease transmission model, the rumor propagation model is established. In the basic rumor propagation model, the population is divided into three categories: Ignorants (people who do not know the rumors), Spreaders (people who 
know and spread rumors) and Removers (people who know rumors but do not spread them). Early studies on rumor propagation took the classical Daley-Kendall (DK) model in [1,2] and Maki-Thomson (MT) model in [3] as examples, which were applicable for the phenomenon of rumor propagation in small-scale social networks with direct contact. In the DK model, the authors assumed that the rumor was spread through the contact of rumor spreaders with other groups in the population. Maki and Thomson [3] modified the DK model and proposed that rumors were spread through two-way contact between the rumormongers and other people in the crowd, thus deriving the MT model.

In large-scale social networks, the probability of rumors spreading among individuals is diverse, and the dynamic behaviors of rumors spreading are also varied. Therefore, the study of rumors spreading mainly explores the laws between them. Zanette [4] was the first one to apply the theory of complex networks to analyze the rumor propagation, and then he studied the MT model with small-world network under dynamic and static conditions in [5], in which he found that the network structure would have a momentous impact on rumor propagation. Moreno [6] studied the rumor propagation in homogeneous and heterogeneous networks, respectively, and gave the mean field equation of rumor propagation in homogeneous and scale-free networks. Later, there are an increasing number of scholars [711] to study the rumor spreading issues in complex networks. Kawach [12] pointed out that the memory mechanism of the stifler would affect the spread of rumors and change the spread rate in the process of spreading. Zhao [13] studied the rumor propagation model with forgetting and memory mechanisms. The rumor propagation models with rational consciousness [14], psychological differences [15,16], media reports [17], official refutation [18] and other factors were also considered. In addition, since individuals may not respond to rumors in time, $[19,20]$ studied the rumor propagation models with contact lag between susceptible and infected people. Since various individuals have distinct abilities to spread and absorb information, it is of greater practical significance to study the rumor propagation model [21-24] under heterogeneous networks. In [25], a rumor propagation model based on heterogeneous network was proposed, and two control strategies not only show a good resistance to rumor propagation, but also can effectively reduce the cost of suppressing rumor. In [26], based on a scale-free network, the authors considered the network topology with psychological factors and applied such control strategies as unified immune control, modular surface control and optimal immune control to inhibit the spread of rumors.

For the same rumor, people can choose different languages to spread. In this multilingual environment, diverse languages will increase the complexity of rumor propagation. At present, some achievements have been made for rumor propagation in a multilingual environment. Using multiple-group epidemic model research techniques, Wang in [27] first proposed a SIR rumor propagation model in a multilingual environment, in which all individuals were divided into different groups according to distinct languages, and a new model with a cross-propagation mechanism was established. Different from Wang's model, Li [28] established and analyzed the I2S2R rumor propagation model in the complex network and discussed the dynamics through the mean field equation. However, up to now, there is no study on rumor propagation model with heterogeneous networks in a multilingual environment.

Motivated by the above works, in this paper, we study the propagation and control of rumors with heterogeneous networks in a multilingual environment. The contributions of this paper are as follows: (1) In the multilingual environment, this paper considers the influence of immunologic mechanism on the ignorant and proposes a new I2SR rumor propagation model. (2) By using next-generation matrix method and Lyapunov stability theory, the existence and global stability of rumor-free equilibrium and rumor equilibrium are analyzed. (3) In order to effectively suppress the spread of the rumor, a real-time control method on the spreaders is proposed, which can exterminate the rumor in a short time and greatly shorten the time of rumor spread. (4) It is challenging and sometimes impossible to consume limited resources to suppress rumor spreading continuously. Therefore, we propose an event-triggered impulsive control strategy. By applying the corresponding event-triggered impulsive control mechanism to the spreaders, we can effectively save control costs and guarantee the extinction of rumor.

The rest of this paper is organized as follows. In Sect. 2, we introduce the I2SR rumor propagation model and prove the positivity of the solution. In Sect. 3, we investigate the dynamic behaviors of the model and analyze the existence and global stability of rumor-free equilibrium and rumor equilibrium, 
respectively. In Sect. 4, the dynamics of rumor propagation under continuous control is studied. In Sect. 5, the dynamics of rumor propagation under the eventtriggered impulsive control is investigated. In Sect. 6, we present some numerical simulations. In Sect. 7, the conclusion is given.

\section{Network model}

In this paper, we consider the rumor propagation dynamics over heterogeneous networks in a multilingual environment. We propose four states to indicate the different status of users in the process of rumor spreading. Ignorants $(I(t))$ mean the people who do not know the rumor. Spreaders $1\left(S^{1}(t)\right)$ represent the people who know and spread the rumor in the first language. Spreaders $2\left(S^{2}(t)\right)$ mean the people who know and spread the rumor in the second language. Removers $(R(t))$ mean the people who know the rumor but do not spread it.

The whole population and their communication are described by a network. An individual is represented by a node, and the potential communication between two nodes is represented by an edge, along which rumors may spread. The degree of a node is the number of all its connected edges. Without loss of generality, we assume the people in a network are divided into $n$ groups in view of their degrees, in which people with same degree are assigned to the same group. Let $I_{k_{i}}(t), S_{k_{i}}^{1}(t), S_{k_{i}}^{2}(t)$ and $R_{k_{i}}(t)$ denote the densities of Ignorants, Spreaders 1 , Spreaders 2 and Removers with $k_{i}$ degree in group $i(i=1, \ldots, n)$ at time $t$, respectively. The active nodes in the same group satisfy $I_{k_{i}}(t)+S_{k_{i}}^{1}(t)+S_{k_{i}}^{2}(t)+$ $R_{k_{i}}(t)=1$. In order to depict the process of rumor spreading, we further assume that the criteria for rumor propagation are as follows.

(1) In the process of rumor propagation, the probability of an ignorant becoming a spreader is associated with its degree. Therefore, rumor propagation law is related to the degree of the individual, which is more consistent with the law of rumor propagation in the actual situation. An ignorant becomes $S_{k_{i}}^{v}(\nu=1,2)$ if it believes the rumor, and the rumor conversion rates are $\alpha\left(k_{i}\right)(v=1)$ and $\rho\left(k_{i}\right)(v=2)$, respectively. An ignorant is connected to one or more $S_{k_{i}}^{v}(v=1,2)$ with probability $\Phi_{1}(t)(v=1)$ or $\Phi_{2}(t)(v=2)$ at time $t$. Thus, the infected probability for an ignorant is $\alpha\left(k_{i}\right) \Phi_{1}(t)$ or $\rho\left(k_{i}\right) \Phi_{2}(t)$ at time $t$. The probability of $S_{k_{i}}^{1}(t)$ converting to $S_{k_{i}}^{2}(t)$ is $\gamma$.

(2) In the rumor spreading, some ignorants are immune to the rumor and transform into $R_{k_{i}}(t)$ with probability $\mu$. The spreaders $S_{k_{i}}^{v}(t)(v=1,2)$ are cured and transformed into $R_{k_{i}}(t)$ with probability $\beta_{1}(v=1)$ or $\beta_{2}(v=2)$. The new ignorants join the network with rate $p$ at any time $t$. The active nodes lose interest in rumor spreading with rate $d$ at any time.

Based on the above analysis, the process of rumor propagation is shown in Fig. 1, and the dynamics of rumor spreading model with heterogeneous networks in a multilingual environment is given by:

$$
\left\{\begin{aligned}
\frac{\mathrm{d} I_{k_{i}}(t)}{\mathrm{d} t}= & p-\alpha\left(k_{i}\right) I_{k_{i}}(t) \Phi_{1}(t) \\
& -\rho\left(k_{i}\right) I_{k_{i}}(t) \Phi_{2}(t)-(\mu+d) I_{k_{i}}(t) \\
\frac{\mathrm{d} S_{k_{i}}^{1}(t)}{\mathrm{d} t}= & \alpha\left(k_{i}\right) I_{k_{i}}(t) \Phi_{1}(t)-\beta_{1} S_{k_{i}}^{1}(t)-(\gamma+d) S_{k_{i}}^{1}(t) \\
\frac{\mathrm{d} S_{k_{i}}^{2}(t)}{\mathrm{d} t}= & \rho\left(k_{i}\right) I_{k_{i}}(t) \Phi_{2}(t)-\beta_{2} S_{k_{i}}^{2}(t) \\
& +\gamma S_{k_{i}}^{1}(t)-d S_{k_{i}}^{2}(t) \\
\frac{\mathrm{d} R_{k_{i}}(t)}{\mathrm{d} t}= & \beta_{1} S_{k_{i}}^{1}(t)+\beta_{2} S_{k_{i}}^{2}(t)+\mu I_{k_{i}}(t)-d R_{k_{i}}(t)
\end{aligned}\right.
$$

where $i=1,2, \ldots, n, t \geq 0 . \Phi_{v}(t)(v=1,2)$ represent the probabilities that an ignorant could get in touch with Spreaders 1 and Spreaders 2, respectively. The $\Phi_{\nu}(t)$ is defined as

$\Phi_{\nu}(t)=\frac{\sum_{i=1}^{n} \theta\left(k_{i}\right) Z\left(k_{i}\right) S_{k_{i}}^{\nu}(t)}{\langle k\rangle}$

where $Z\left(k_{i}\right)$ is the probability of a person with degree $k_{i}$, which equals the ratio of people with degree $k_{i}$ to

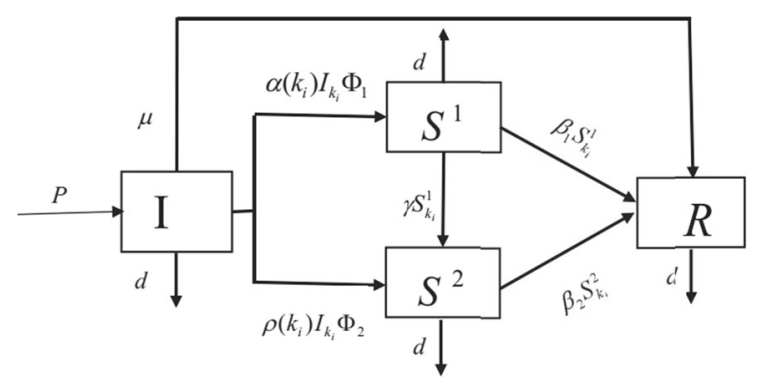

Fig. 1 The state transition diagram of I2SR model 
whole people in the network. Hence, $\sum_{i=1}^{n} Z\left(k_{i}\right)=1$. $\langle k\rangle=\sum_{i=1}^{n} k_{i} Z\left(k_{i}\right)$ is the average degree of the node in the network, and $\theta\left(k_{i}\right)$ measures the infectivity of a person with degree $k_{i}$. At any time $t$, the active nodes in the network satisfy $I_{k_{i}}(t)+S_{k_{i}}^{1}(t)+S_{k_{i}}^{2}(t)+R_{k_{i}}(t)=1$.

Using $I_{k_{i}}(t)=1-S_{k_{i}}^{1}(t)-S_{k_{i}}^{2}(t)-R_{k_{i}}(t)$, model (1) is described by

$$
\left\{\begin{aligned}
\frac{\mathrm{d} S_{k_{i}}^{1}(t)}{\mathrm{d} t}= & \alpha\left(k_{i}\right)\left(1-S_{k_{i}}^{1}(t)-S_{k_{i}}^{2}(t)-R_{k_{i}}(t)\right) \Phi_{1}(t) \\
& -\beta_{1} S_{k_{i}}^{1}(t)-(\gamma+d) S_{k_{i}}^{1}(t) \\
\frac{\mathrm{d} S_{k_{i}}^{2}(t)}{\mathrm{d} t}= & \rho\left(k_{i}\right)\left(1-S_{k_{i}}^{1}(t)-S_{k_{i}}^{2}(t)-R_{k_{i}}(t)\right) \Phi_{2}(t) \\
& -\left(\beta_{2}+d\right) S_{k_{i}}^{2}(t)+\gamma S_{k_{i}}^{1}(t) \\
\frac{\mathrm{d} R_{k_{i}}(t)}{\mathrm{d} t}= & \beta_{1} S_{k_{i}}^{1}(t)+\beta_{2} S_{k_{i}}^{2}(t) \\
& +\mu\left(1-S_{k_{i}}^{1}(t)-S_{k_{i}}^{2}(t)-R_{k_{i}}(t)\right)-d R_{k_{i}}(t) .
\end{aligned}\right.
$$

It is easy to verify that the region $\mathcal{M}=\left\{\left(S_{k_{i}}^{1}(t), S_{k_{i}}^{2}(t)\right.\right.$, $\left.R_{k_{i}}(t)\right) \in \mathbb{R}_{+}^{3 n} \mid S_{k_{i}}^{1}(t)+S_{k_{i}}^{2}(t)+R_{k_{i}}(t) \leq 1, k=$ $1,2, \ldots, n\}$ is a positive invariant set for model (2). As a matter of convenience, let $\mathcal{M}=\left\{\left(x_{i}(t), y_{i}(t), z_{i}(t)\right) \in\right.$ $\left.\mathbb{R}_{+}^{3 n} \mid x_{i}(t)+y_{i}(t)+z_{i}(t) \leq 1, i=1,2, \ldots, n\right\}$ be the meaningful physical domain for model (2).

Remark 1 In [27,28], the authors studied the problems of rumor spreading in a multilingual environment, but the network is homogeneous. In fact, the social networks are always heterogeneous. Therefore, we propose a new propagation model with heterogeneous networks and consider the immune mechanism of the ignorants in this paper.

Next, the following useful lemma is necessary.

Lemma 1 The set $\mathcal{M}$ is positively invariant to model (2).

Proof Denote $\chi(t)=\left(\chi_{1}(t), \chi_{2}(t), \ldots, \chi_{n}(t)\right)^{T}$, where $\chi_{i}(t)=\left(x_{i}(t), y_{i}(t), z_{i}(t)\right)$ for $i=1,2, \ldots, n$; then, model (2) can be rewritten as

$$
\frac{\mathrm{d} \chi_{i}(t)}{\mathrm{d} t}=f_{i}\left(x_{i}(t)\right)
$$

where $f_{i}\left(x_{i}(t)\right)=$ $\left(\begin{array}{c}\alpha\left(k_{i}\right)\left(1-x_{i}(t)-y_{i}(t)-z_{i}(t)\right) \Phi_{1}-\beta_{1} x_{i}(t)-(\gamma+d) x_{i}(t) \\ \rho\left(k_{i}\right)\left(1-x_{i}(t)-y_{i}(t)-z_{i}(t)\right) \Phi_{2}-\left(\beta_{2}+d\right) y_{i}(t)+\gamma x_{i}(t) \\ \beta_{1} x_{i}(t)+\beta_{2} y_{i}(t)+\mu\left(1-x_{i}(t)-y_{i}(t)-z_{i}(t)\right)-d z_{i}(t)\end{array}\right)$. Note that $\mathcal{M}$ is a bounded compact set. We need to prove that $\chi(t) \in \mathcal{M}$ for all $t \geq 0$. Denote the boundary of $\mathcal{M}$ as $\partial \mathcal{M}$; then, it includes the following $3 n$ sets:

$\partial \mathcal{M}_{1}=\left\{\chi \in \mathcal{M} \mid x_{i}(t)=0\right\}$,

$\partial \mathcal{M}_{2}=\left\{\chi \in \mathcal{M} \mid y_{i}(t)=0\right\}$,

$\partial \mathcal{M}_{3}=\left\{\chi \in \mathcal{M} \mid z_{i}(t)=0\right\}$,

$\partial \mathcal{M}_{4}=\left\{\chi \in \mathcal{M} \mid x_{i}(t)+y_{i}(t)+z_{i}(t)=1\right\}$,

which have outer normal vectors as follows:

$$
\begin{aligned}
& \varphi_{i}^{1}=(0, \ldots, 0, \underbrace{-1}_{i}, 0, \ldots, 0), \\
& \varphi_{i}^{2}=(0, \ldots, 0, \underbrace{-1}_{i+n}, 0, \ldots, 0), \\
& \varphi_{i}^{3}=(0, \ldots, 0, \underbrace{-1}_{i+2 n}, 0, \ldots, 0), \\
& \varphi_{i}^{4}=(0, \ldots, 0, \underbrace{1}_{i}, 0, \ldots, \\
& 0, \underbrace{1}_{i+n}, 0, \ldots, 0, \underbrace{1}_{i+2 n}, 0, \ldots, 0) .
\end{aligned}
$$

Through certain calculation, we have

$$
\begin{aligned}
& \left\langle\left.\frac{\mathrm{d} \chi(t)}{\mathrm{d} t}\right|_{\chi \in \partial \mathcal{M}_{1}, \varphi_{i}^{1}}\right\rangle=-\alpha\left(k_{i}\right)\left(1-y_{i}(t)-z_{i}(t)\right) \\
& \frac{\sum_{j \neq i}^{n} \theta\left(k_{i}\right) Z\left(k_{i}\right) x_{i}(t)}{\langle k\rangle} \leq 0, \\
& \left\langle\left.\frac{\mathrm{d} \chi(t)}{\mathrm{d} t}\right|_{\chi \in \partial \mathcal{M}_{2}, \varphi_{i}^{2}}\right\rangle=-\rho\left(k_{i}\right)\left(1-x_{i}(t)-z_{i}(t)\right) \\
& \frac{\sum_{j \neq i}^{n} \theta\left(k_{i}\right) Z\left(k_{i}\right) y_{i}(t)}{\langle k\rangle} \leq 0, \\
& \left\langle\left.\frac{\mathrm{d} \chi(t)}{\mathrm{d} t}\right|_{\chi \in \partial \mathcal{M}_{3}, \varphi_{i}^{3}}\right\rangle \\
& =-\left[\beta_{1} x_{i}(t)+\beta_{2} y_{i}(t)+\mu\left(1-x_{i}(t)-y_{i}(t)\right)\right] \leq 0, \\
& \left\langle\left.\frac{\mathrm{d} \chi(t)}{\mathrm{d} t}\right|_{\chi \in \partial \mathcal{M}_{4}, \varphi_{i}^{4}}\right\rangle=-\left(x_{i}(t)+y_{i}(t)+z_{i}(t)\right) \leq 0 .
\end{aligned}
$$

Thus, the claimed result can be obtained directly from Lemma 2 in [29]. The proof is completed.

\section{Existence of the equilibrium solution}

In this section, we will analyze the dynamics of model (1) and give some conditions for rumor extinction or persistence.

As far as we know, whether the rumor breaks out is dependent on the basic reproduction number $r_{0}$. For the 
calculation of $r_{0}$, the next-generation matrix method is used.

In order to facilitate the calculation of basic reproduction number of model (1), we let $\psi=\left(S_{k_{i}}^{1}(t)\right.$, $\left.S_{k_{i}}^{2}(t), I_{k_{i}}(t), R_{k_{i}}(t)\right)^{T}$, model (1) can be rewritten as follows:

$\frac{\mathrm{d} \psi}{\mathrm{d} t}=\mathcal{F}(\psi)-\mathcal{V}(\psi)$

where $\mathcal{F}(\psi)=\left(\begin{array}{c}\alpha\left(k_{i}\right) I_{k_{i}}(t) \Phi_{1}(t) \\ \rho\left(k_{i}\right) I_{k_{i}}(t) \Phi_{2}(t) \\ 0 \\ 0\end{array}\right)$

$$
\begin{aligned}
& r_{01}=\frac{p \sum_{i=1}^{n} \alpha\left(k_{i}\right) \theta\left(k_{i}\right) Z\left(k_{i}\right)}{\langle k\rangle(d+\mu)\left(\beta_{1}+\gamma+d\right)}, \\
& r_{02}=\frac{p \sum_{i=1}^{n} \rho\left(k_{i}\right) \theta\left(k_{i}\right) Z\left(k_{i}\right)}{\langle k\rangle(d+\mu)\left(\beta_{2}+d\right)} .
\end{aligned}
$$

$\mathcal{V}(\psi)=\left(\begin{array}{c}\beta_{1} S_{k_{i}}^{1}(t)+(\gamma+d) S_{k_{i}}^{1}(t) \\ \beta_{2} S_{k_{i}}^{2}(t)-\gamma S_{k_{i}}^{1}(t)+d S_{k_{i}}^{2}(t) \\ \alpha\left(k_{i}\right) I_{k_{i}}(t) \Phi_{1}(t)+\rho\left(k_{i}\right) I_{k_{i}}(t) \Phi_{2}(t)+(d+\mu) I_{k_{i}}(t)-p \\ -\beta_{1} S_{k_{i}}^{1}(t)-\beta_{2} S_{k_{i}}^{2}(t)-\mu I_{k_{i}}(t)+d R_{k_{i}}(t)\end{array}\right)$.

It is obvious that $E_{0}=\left\{\left(\frac{p}{d+\mu}, 0,0,1-\frac{p}{d+\mu}\right), \ldots\right.$, $\left.\left(\frac{p}{d+\mu}, 0,0,1-\frac{p}{d+\mu}\right)\right\}$ is a rumor-free equilibrium point of model (1). The Jacobian matrices of $\mathcal{F}(\psi)$ and $\mathcal{V}(\psi)$ at $E_{0}=\left\{\left(\frac{p}{d+\mu}, 0,0,1-\frac{p}{d+\mu}\right), \ldots,\left(\frac{p}{d+\mu}, 0,0,1-\right.\right.$ $\left.\left.\frac{p}{d+\mu}\right)\right\}$ are

$\mathcal{D} \mathcal{F}\left(E_{0}\right)=\left(\begin{array}{cc}F & \mathbf{0} \\ \mathbf{0} & \mathbf{0}\end{array}\right), \quad \mathcal{D} \mathcal{V}\left(E_{0}\right)=\left(\begin{array}{cc}V & \mathbf{0} \\ J_{1} & J_{2}\end{array}\right)$

where $F=\left(\begin{array}{cc}F_{1} & \mathbf{0} \\ \mathbf{0} & F_{2}\end{array}\right), V=\left(\begin{array}{cc}\left(\beta_{1}+\gamma+d\right) \mathcal{I} & \mathbf{0} \\ -\gamma \mathcal{I} & \left(\beta_{2}+d\right) \mathcal{I}\end{array}\right)$,
From this, the following theorem is given.

Theorem 1 For the basic reproduction number $r_{0}$, the following three cases hold.

Case 1. If $r_{0}<1$, model (1) shows a rumor free equilibrium $E_{0}=\left\{\left(\frac{p}{d+\mu}, 0,0,1-\frac{p}{d+\mu}\right), \ldots,\left(\frac{p}{d+\mu}, 0,0,1-\right.\right.$ $\left.\left.\frac{p}{d+\mu}\right)\right\}$.

Case 2. If $r_{01}<1$ and $r_{02}>1$, model (1) shows a unique equilibrium point $E_{1}^{*}\left(I_{1 k_{i}}^{*}, S_{1 k_{i}}^{1 *}, S_{1 k_{i}}^{2 *}, R_{1 k_{i}}^{*}\right)$ where

$$
\begin{aligned}
F_{1}= & \left(\begin{array}{cccc}
\frac{\alpha\left(k_{1}\right) I_{k_{1}}(t) \theta\left(k_{1}\right) Z\left(k_{1}\right)}{\langle k\rangle} & \frac{\alpha\left(k_{1}\right) I_{k_{1}}(t) \theta\left(k_{2}\right) Z\left(k_{2}\right)}{\langle k\rangle} & \cdots & \frac{\alpha\left(k_{1}\right) I_{k_{1}}(t) \theta\left(k_{n}\right) Z\left(k_{n}\right)}{\langle k\rangle} \\
\frac{\alpha\left(k_{2}\right) I_{k_{2}}(t) \theta\left(k_{1}\right) Z\left(k_{1}\right)}{\langle k\rangle} & \frac{\alpha\left(k_{2}\right) I_{k_{2}}(t) \theta\left(k_{2}\right) Z\left(k_{2}\right)}{\langle k\rangle} & \cdots & \frac{\alpha\left(k_{2}\right) I_{k_{2}}(t) \theta\left(k_{n}\right) Z\left(k_{n}\right)}{\langle k\rangle} \\
\vdots & \vdots & \ddots & \vdots \\
\frac{\alpha\left(k_{n}\right) I_{k_{n}}(t) \theta\left(k_{1}\right) Z\left(k_{1}\right)}{\langle k\rangle} & \frac{\alpha\left(k_{n}\right) I_{k_{n}}(t) \theta\left(k_{2}\right) Z\left(k_{2}\right)}{\langle k\rangle} & \cdots & \frac{\alpha(n) I_{k_{n}}(t) \theta\left(k_{n}\right) Z\left(k_{n}\right)}{\langle k\rangle}
\end{array}\right), \\
F_{2}= & \left(\begin{array}{cccc}
\frac{\rho\left(k_{1}\right) I_{k_{1}}(t) \theta\left(k_{1}\right) Z\left(k_{1}\right)}{\langle k\rangle} & \frac{\rho\left(k_{1}\right) I_{k_{1}}(t) \theta\left(k_{2}\right) Z\left(k_{2}\right)}{\langle k\rangle} & \cdots & \frac{\rho\left(k_{1}\right) I_{k_{1}}(t) \theta\left(k_{n}\right) Z\left(k_{n}\right)}{\langle k\rangle} \\
\frac{\rho\left(k_{2}\right) I_{k_{2}}(t) \theta\left(k_{1}\right) Z\left(k_{1}\right)}{\langle k\rangle} & \frac{\rho\left(k_{2}\right) I_{k_{2}}(t) \theta\left(k_{2}\right) Z\left(k_{2}\right)}{\langle k\rangle} & \cdots & \frac{\rho\left(k_{2}\right) I_{k_{2}}(t) \theta\left(k_{n}\right) Z\left(k_{n}\right)}{\langle k\rangle} \\
\vdots & \vdots & \ddots & \vdots \\
\frac{\rho\left(k_{n}\right) I_{k_{n}}(t) \theta\left(k_{1}\right) Z\left(k_{1}\right)}{\langle k\rangle} & \frac{\rho\left(k_{n}\right) I_{k_{n}}(t) \theta\left(k_{2}\right) Z\left(k_{2}\right)}{\langle k\rangle} & \cdots & \frac{\rho(n) I_{k_{n}}(t) \theta\left(k_{n}\right) Z\left(k_{n}\right)}{\langle k\rangle}
\end{array}\right), \\
J_{1}= & \left(\begin{array}{c}
F_{1} \\
F_{2} \\
-\beta_{1} \mathcal{I}-\beta_{2} \mathcal{I}
\end{array}\right), \\
J_{2}= & \left(\begin{array}{c}
(d+\mu) \mathcal{I} \mathbf{0} \\
-\mu \mathcal{I} \quad d \mathcal{I}
\end{array}\right),
\end{aligned}
$$

and $\mathcal{I}$ is a unit matrix with appropriate dimension. 
$S_{1 k_{i}}^{1 *}=0, S_{1 k_{i}}^{2 *}=\frac{p \rho\left(k_{i}\right) \Phi_{2}^{*}}{\left[\rho\left(k_{i}\right) \Phi_{2}^{*}+d+\mu\right]\left(\beta_{2}+d\right)}$,

$I_{1 k_{i}}^{*}=\frac{\left(d+\beta_{2}\right) S_{1 k_{i}}^{2 *}}{\rho\left(k_{i}\right) \Phi_{2}^{*}}, R_{1 k_{i}}^{*}=\frac{\beta_{2} S_{1 k_{i}}^{2 *}+\mu I_{1 k_{i}}^{*}}{d}$,

$\Phi_{2}^{*}=\frac{\sum_{i=1}^{n} \theta\left(k_{i}\right) Z\left(k_{i}\right) S_{1 k_{i}}^{2 *}}{\langle k\rangle}$.

Case 3. If $r_{01}>1$ and $r_{02}>1$, the unique positive rumor equilibrium point of model (1) is $E_{2}^{*}\left(I_{2 k_{i}}^{*}, S_{2 k_{i}}^{1 *}, S_{2 k_{i}}^{2 *}, R_{2 k_{i}}^{*}\right)$ where

$S_{2 k_{i}}^{1 *}=\frac{p \alpha\left(k_{i}\right) \Phi_{1}^{*}}{\left[\alpha\left(k_{i}\right) \Phi_{1}^{*}+\rho\left(k_{i}\right) \Phi_{2}^{*}+d+\mu\right]\left(\beta_{1}+\gamma+d\right)}$,

$S_{2 k_{i}}^{2 *}=\frac{p \rho\left(k_{i}\right)\left(\beta_{1}+\gamma+d\right) \Phi_{2}^{*}+\gamma p \alpha\left(k_{i}\right) \Phi_{1}^{*}}{\left[\alpha\left(k_{i}\right) \Phi_{1}^{*}+\rho\left(k_{i}\right) \Phi_{2}^{*}+d+\mu\right]\left(\beta_{1}+\gamma+d\right)\left(\beta_{2}+d\right)}$,

$I_{2 k_{i}}^{*}=\frac{p}{\alpha\left(k_{i}\right) \Phi_{1}^{*}+\rho\left(k_{i}\right) \Phi_{2}^{*}+d+\mu}$,

$R_{2 k_{i}}^{*}=\frac{\beta_{1} S_{2 k_{i}}^{1 *}+\beta_{2} S_{2 k_{i}}^{2 *}+\mu I_{2 k_{i}}^{*}}{d}$,

$\Phi_{1}^{*}=\frac{\sum_{i=1}^{n} \theta\left(k_{i}\right) Z\left(k_{i}\right) S_{2 k_{i}}^{1 *}}{\langle k\rangle}$.

Proof For the case $r_{0}<1$, it is easy to get that the rumor-free equilibrium point $E_{0}$. In model (1), we denote the equilibrium solution by $E^{*}=\left\{\left(I_{k_{1}}^{*}, S_{k_{1}}^{1 *}\right.\right.$, $\left.\left.S_{k_{1}}^{2 *}, R_{k_{1}}^{*}\right), \ldots,\left(I_{k_{n}}^{*}, S_{k_{n}}^{1 *}, S_{k_{n}}^{2 *}, R_{k_{n}}^{*}\right)\right\}$. Then, it should satisfy

$p-\alpha\left(k_{i}\right) I_{k_{i}}^{*} \Phi_{1}^{*}-\rho\left(k_{i}\right) I_{k_{i}}^{*} \Phi_{2}^{*}-(\mu+d) I_{k_{i}}^{*}=0$

$\alpha\left(k_{i}\right) I_{k_{i}}^{*} \Phi_{1}^{*}-\beta_{1} S_{k_{i}}^{1 *}-(\gamma+d) S_{k_{i}}^{1 *}=0$

$\rho\left(k_{i}\right) I_{k_{i}}^{*} \Phi_{2}^{*}-\beta_{2} S_{k_{i}}^{2 *}+\gamma S_{k_{i}}^{1 *}-d S_{k_{i}}^{2 *}=0$

$\beta_{1} S_{k_{i}}^{1 *}+\beta_{2} S_{k_{i}}^{2 *}+\mu I_{k_{i}}^{*}-d R_{k_{i}}^{*}=0$.

For the case of $r_{01}<1$ and $r_{02}>1$, it has

$$
\begin{aligned}
\Phi_{2}^{*} & =\frac{\sum_{i=1}^{n} \theta\left(k_{i}\right) Z\left(k_{i}\right) S_{1 k_{i}}^{2 *}}{\langle k\rangle} \\
& =\frac{1}{\langle k\rangle} \sum_{i=1}^{n} \frac{p \rho\left(k_{i}\right) \theta\left(k_{i}\right) Z\left(k_{i}\right) \Phi_{2}^{*}}{\left[\rho\left(k_{i}\right) \Phi_{2}^{*}+d+\mu\right]\left(\beta_{2}+d\right)} .
\end{aligned}
$$

According to simple calculation, it has

$\Phi_{2}^{*}\left(1-\frac{1}{\langle k\rangle} \sum_{i=1}^{n} \frac{p \rho\left(k_{i}\right) \theta\left(k_{i}\right) Z\left(k_{i}\right)}{\left[\rho\left(k_{i}\right) \Phi_{2}^{*}+d+\mu\right]\left(\beta_{2}+d\right)}\right)=0$.
Let

$G\left(\Phi_{2}^{*}\right)=1-\frac{1}{\langle k\rangle} \sum_{i=1}^{n} \frac{p \rho\left(k_{i}\right) \theta\left(k_{i}\right) Z\left(k_{i}\right)}{\left[\rho\left(k_{i}\right) \Phi_{2}^{*}+d+\mu\right]\left(\beta_{2}+d\right)}$.

Since $G^{\prime}\left(\Phi_{2}^{*}\right)>0$ for all $\Phi_{2}^{*}$ and $\lim _{\Phi_{2}^{*} \rightarrow \infty} G\left(\Phi_{2}^{*}\right)=$ 1 , then $G\left(\Phi_{2}^{*}\right)=0$ if and only if $\lim _{\Phi_{2}^{*} \rightarrow 0^{+}} G\left(\Phi_{2}^{*}\right)<$ 0 . That means model (1) has an equilibrium solution $E_{1}^{*}$ if and only if $r_{01}<1$ and $r_{02}>1$.

For the case of $r_{01}>1$ and $r_{02}>1$, we have

$$
\begin{aligned}
\Phi_{1}^{*} & =\frac{\sum_{i=1}^{n} \theta\left(k_{i}\right) Z\left(k_{i}\right) S_{2 k_{i}}^{1 *}}{\langle k\rangle} \\
& =\frac{p}{\langle k\rangle} \sum_{i=1}^{n} \frac{\alpha\left(k_{i}\right) \theta\left(k_{i}\right) Z\left(k_{i}\right) \Phi_{1}^{*}}{\left(\alpha\left(k_{i}\right) \Phi_{1}^{*}+\rho\left(k_{i}\right) \Phi_{2}^{*}+d+\mu\right)\left(\beta_{1}+\gamma+d\right)}, \\
\Phi_{2}^{*} & =\frac{\sum_{i=1}^{n} \theta\left(k_{i}\right) Z\left(k_{i}\right) S_{2 k_{i}}^{2 *}}{\langle k\rangle} \\
& =\frac{p}{\langle k\rangle} \sum_{i=1}^{n} \frac{\rho\left(k_{i}\right) \theta\left(k_{i}\right) Z\left(k_{i}\right) \Phi_{2}^{*}+\gamma \alpha\left(k_{i}\right) \theta\left(k_{i}\right) Z\left(k_{i}\right) \Phi_{1}^{*}}{\left(\alpha\left(k_{i}\right) \Phi_{1}^{*}+\rho\left(k_{i}\right) \Phi_{2}^{*}+d+\mu\right)\left(\beta_{1}+\gamma+d\right)\left(\beta_{2}+d\right)} .
\end{aligned}
$$

Then,

$$
\begin{aligned}
& \Phi_{1}^{*}\left(1-\frac{p}{\langle k\rangle} \sum_{i=1}^{n} \frac{\alpha\left(k_{i}\right) \theta\left(k_{i}\right) Z\left(k_{i}\right)}{\left(\alpha\left(k_{i}\right) \Phi_{1}^{*}+\rho\left(k_{i}\right) \Phi_{2}^{*}+d+\mu\right)\left(\beta_{1}+\gamma+d\right)}\right)=0, \\
& \Phi_{2}^{*}\left(1-\frac{p}{\langle k\rangle} \sum_{i=1}^{n} \frac{\rho\left(k_{i}\right) \theta\left(k_{i}\right) Z\left(k_{i}\right)}{\left(\alpha\left(k_{i}\right) \Phi_{1}^{*}+\rho\left(k_{i}\right) \Phi_{2}^{*}+d+\mu\right)\left(\beta_{1}+\gamma+d\right)\left(\beta_{2}+d\right)}\right. \\
& \left.\quad-\frac{p}{\langle k\rangle} \sum_{i=1}^{n} \frac{\gamma \alpha\left(k_{i}\right) \theta\left(k_{i}\right) Z\left(k_{i}\right) \Phi_{1}^{*}}{\Phi_{2}^{*}\left(\alpha\left(k_{i}\right) \Phi_{1}^{*}+\rho\left(k_{i}\right) \Phi_{2}^{*}+d+\mu\right)\left(\beta_{1}+\gamma+d\right)\left(\beta_{2}+d\right)}\right) \\
& =0 .
\end{aligned}
$$

Let

$$
\begin{aligned}
& G_{1}\left(\Phi_{1}^{*}, \Phi_{2}^{*}\right)=\left(1-\frac{p}{\langle k\rangle} \sum_{i=1}^{n}\right. \\
& \left.\quad \frac{\alpha\left(k_{i}\right) \theta\left(k_{i}\right) Z\left(k_{i}\right)}{\left(\alpha\left(k_{i}\right) \Phi_{1}^{*}+\rho\left(k_{i}\right) \Phi_{2}^{*}+d+\mu\right)\left(\beta_{1}+\gamma+d\right)}\right), \\
& G_{2}\left(\Phi_{1}^{*}, \Phi_{2}^{*}\right) \\
& \quad=\left(1-\frac{p}{\langle k\rangle} \sum_{i=1}^{n} \frac{\rho\left(k_{i}\right) \theta\left(k_{i}\right) Z\left(k_{i}\right)}{\left(\alpha\left(k_{i}\right) \Phi_{1}^{*}+\rho\left(k_{i}\right) \Phi_{2}^{*}+d+\mu\right)\left(\beta_{1}+\gamma+d\right)\left(\beta_{2}+d\right)}\right. \\
& \left.\quad-\frac{p}{\langle k\rangle} \sum_{i=1}^{n} \frac{\gamma \alpha\left(k_{i}\right) \theta\left(k_{i}\right) Z\left(k_{i}\right) \Phi_{1}^{*}}{\Phi_{2}^{*}\left(\alpha\left(k_{i}\right) \Phi_{1}^{*}+\rho\left(k_{i}\right) \Phi_{2}^{*}+d+\mu\right)\left(\beta_{1}+\gamma+d\right)\left(\beta_{2}+d\right)}\right) .
\end{aligned}
$$

Due to $\frac{\partial G_{1}\left(\Phi_{1}^{*}, \Phi_{2}^{*}\right)}{\partial \Phi_{1}^{*}}>0$ for all $\Phi_{1}^{*}$ and $\lim _{\Phi_{1}^{*} \rightarrow \infty}$ $G_{1}\left(\Phi_{1}^{*}, \Phi_{2}^{*}\right)=1$, then $S_{2 k_{i}}^{1 *}>0$ if $\lim _{\Phi_{1}^{*} \rightarrow 0^{+}} G_{1}\left(\Phi_{1}^{*}, 0\right)$ $<0$. Similarly, $S_{2 k_{i}}^{2 *}>0$ if $\lim _{\Phi_{2}^{*} \rightarrow 0^{+}} G_{2}\left(0, \Phi_{2}^{*}\right)<0$. Based on the definition of $r_{01}$ and $r_{02}$, one can conclude that $r_{01}>1$ and $r_{02}>1$. From the above analysis, we 
can get that model (1) has a unique positive solution if and only if $r_{01}>1$ and $r_{02}>1$.

Remark 2 It can be seen that the basic reproduction number $r_{0}$ is dependent on the infection rates $\alpha\left(k_{i}\right)$ and $\rho\left(k_{i}\right)$. In the previous works, the infection rate is a constant which is independent of the degree of the individual. However, the infection rate is related to the degree of the individual in our paper.

Remark 3 From model (1), we can see that the Spreader 1 is transformed into Spreaders 2 with a certain probability $\gamma$, which means that the rumor Spreader 2 will not die out when rumor Spreaders 1 exist. Therefore, our paper discusses two situations of rumor existence. The one is that the Spreaders 1 are extinct and the Spreaders 2 exist. The other is that two kinds of spreaders exist.

Remark 4 Through the analysis of Theorem 1 and the calculation of the basic reproduction number $r_{0}$, we confirm that two kinds of rumor spreaders will be extinct when $r_{0}<1$. But we cannot accurately judge whether there is only one kind of rumor spreaders or both when $r_{0}>1$. For this case, we need to further analyze.

Theorem 2 The rumor-free equilibrium $E_{0}$ of model (1) is locally asymptotically stable if $r_{0}<1$.

Proof According to stability theory [30], the local stability of rumor-free equilibrium $E_{0}$ of model (1) is related to the eigenvalues of the corresponding Jacobian matrix $J\left(E_{0}\right)$. To analyze the local stability of model (1) at $E_{0}$, we firstly derive the Jacobian matrix $J\left(E_{0}\right)$ as follows

$J\left(E_{0}\right)=\left(\begin{array}{cccc}A_{11} & A_{12} & \cdots & A_{1 n} \\ A_{21} & A_{22} & \cdots & A_{2 n} \\ \vdots & \vdots & \ddots & \vdots \\ A_{n 1} & A_{n 2} & \cdots & A_{n n}\end{array}\right)$

where

$$
\begin{aligned}
A_{i i} & =\left(\begin{array}{cccc}
\Lambda_{1} & 0 & 0 & 0 \\
\gamma & \Lambda_{2} & 0 & 0 \\
-\frac{p \alpha\left(k_{i}\right)}{(d+\mu)\langle k\rangle} \theta\left(k_{i}\right) Z\left(k_{i}\right) & \frac{p \rho\left(k_{i}\right)}{(d+\mu)\langle k\rangle} \theta\left(k_{i}\right) Z\left(k_{i}\right) & -(d+\mu) & 0 \\
\beta_{1} & \beta_{2} & \mu & -d
\end{array}\right), \\
A_{i j} & =\left(\begin{array}{cccc}
\frac{p \alpha\left(k_{i}\right)}{(d+\mu)\langle k\rangle} \theta\left(k_{i}\right) Z\left(k_{i}\right) & 0 & 0 & 0 \\
0 & \frac{p \rho\left(k_{i}\right)}{(d+\mu)\langle k\rangle} \theta\left(k_{i}\right) Z\left(k_{i}\right) & 0 & 0 \\
-\frac{p \alpha\left(k_{i}\right)}{(d+\mu)\langle k\rangle} \theta\left(k_{i}\right) Z\left(k_{i}\right) & \frac{p \rho\left(k_{i}\right)}{(d+\mu)\langle k\rangle} \theta\left(k_{i}\right) Z\left(k_{i}\right) & 0 & 0 \\
0 & 0 & 0 & 0
\end{array}\right),
\end{aligned}
$$

$\Lambda_{1}=\frac{p \alpha\left(k_{i}\right)}{(d+\mu)\langle k\rangle} \theta\left(k_{i}\right) Z\left(k_{i}\right)-\beta_{1}-\gamma-d$ and $\Lambda_{2}=$ $\frac{p \rho\left(k_{i}\right)}{(d+\mu)\langle k\rangle} \theta\left(k_{i}\right) Z\left(k_{i}\right)-\beta_{2}-d$.

The characteristic equation of $J\left(E_{0}\right)$ is

$$
\begin{aligned}
& \left(\lambda+\beta_{1}+\gamma+d\right)^{n-1}\left(1-\frac{p \sum_{i=1}^{n} \alpha\left(k_{i}\right) \theta\left(k_{i}\right) Z\left(k_{i}\right)}{\langle k\rangle(d+\mu)\left(\lambda+\beta_{1}+\gamma+d\right)}\right) \\
& \left(\lambda+\beta_{2}+d\right)^{n-1} \times\left(1-\frac{p \sum_{i=1}^{n} \rho\left(k_{i}\right) \theta\left(k_{i}\right) Z\left(k_{i}\right)}{\langle k\rangle(d+\mu)\left(\lambda+\beta_{2}+d\right)}\right) \\
& (\lambda-(d+\mu))^{n}(\lambda-d)^{n}=0 .
\end{aligned}
$$

The eigenvalues of $J\left(E_{0}\right)$ are given by

$$
\begin{aligned}
\lambda_{1} & =-\left(\beta_{1}+\gamma+d\right), \\
\lambda_{2} & =-\left(\beta_{2}+d\right), \lambda_{3}=-d, \lambda_{4}=-(\mu+d), \\
\lambda_{5} & =\left(\beta_{1}+\gamma+d\right)\left(\frac{p \sum_{i=1}^{n} \alpha\left(k_{i}\right) \theta\left(k_{i}\right) Z\left(k_{i}\right)}{\langle k\rangle(d+\mu)\left(\beta_{1}+\gamma+d\right)}-1\right) \\
& =\left(\beta_{1}+\gamma+d\right)\left(r_{01}-1\right), \\
\lambda_{6} & =\left(\beta_{2}+d\right)\left(\frac{p \sum_{i=1}^{n} \rho\left(k_{i}\right) \theta\left(k_{i}\right) Z\left(k_{i}\right)}{\langle k\rangle(d+\mu)\left(\beta_{2}+d\right)}-1\right) \\
& =\left(\beta_{2}+d\right)\left(r_{02}-1\right),
\end{aligned}
$$

where $\lambda_{1}$ and $\lambda_{2}$ with multiplicity $n-1, \lambda_{3}$ and $\lambda_{4}$ with multiplicity $n$, respectively. $\lambda_{5}$ and $\lambda_{6}$ are simple eigenvalues. We can easily see that $\lambda_{1}, \lambda_{2}, \lambda_{3}$ and $\lambda_{4}$ are negative. Hence, the local stability of $E_{0}$ is determined by $\lambda_{5}$ and $\lambda_{6}$. Since $r_{0}<1, \lambda_{5}$ and $\lambda_{6}$ are negative. Therefore, the rumor-free equilibrium $E_{0}$ of model (1) is locally asymptotically stable.

Lemma 2 [31] For any $a>0$ and $b>0$, if $\frac{d x(t)}{d t} \geq b-a x(t)$ for $t \geq 0$ and $x(0)>0$, we have $\lim _{t \rightarrow \infty} \inf x(t) \geq \frac{b}{a}$; if $\frac{d x(t)}{d t} \leq b-a x(t)$ for $t \geq 0$ and $x(0)>0$, we have $\lim _{t \rightarrow \infty} \sup x(t) \leq \frac{b}{a}$.

Theorem 3 The rumor-free equilibrium $E_{0}$ of model (1) is globally asymptotically stable if $r_{0}<1$.

Proof Based on model (1), we can get that

$$
\begin{aligned}
& \frac{\mathrm{d} I_{k_{i}}(t)}{\mathrm{d} t} \\
& \quad=p-\alpha\left(k_{i}\right) I_{k_{i}}(t) \Phi_{1}(t) \\
& \quad-\rho\left(k_{i}\right) I_{k_{i}}(t) \Phi_{2}(t)-(\mu+d) I_{k_{i}}(t) \\
& \quad \leq p-(\mu+d) I_{k_{i}}(t) .
\end{aligned}
$$

By Lemma 2, one has

$$
\lim _{t \rightarrow \infty} \sup I_{k_{i}}(t) \leq \frac{p}{\mu+d}=: I_{k_{i}}^{0} \text {. }
$$


Thus, for arbitrarily enough small $\varepsilon_{1}>0$, there exist $t_{1}>0$ such that $I_{k_{i}}(t) \leq I_{k_{i}}^{0}+\varepsilon_{1}$ for $t>t_{1}$. When $t>t_{1}$, it has that

$$
\begin{aligned}
\frac{\mathrm{d} S_{k_{i}}^{1}(t)}{\mathrm{d} t} & \leq \alpha\left(k_{i}\right) \Phi_{1}(t)\left(I_{k_{i}}^{0}+\varepsilon_{1}\right) \\
& -\left(\beta_{1}+\gamma+d\right) S_{k_{i}}^{1}(t) .
\end{aligned}
$$

According to the comparison system with the condition $Q_{k_{i}}^{1}(0)=S_{k_{i}}^{1}(0)>0$, let $\tilde{\Phi}_{1}(t)=\frac{\sum_{i=1}^{n} \theta\left(k_{i}\right) Z\left(k_{i}\right) Q_{k_{i}}^{1}(t)}{\langle k\rangle}$, then one has

$$
\begin{aligned}
\frac{\mathrm{d} Q_{k_{i}}^{1}(t)}{\mathrm{d} t} & =\alpha\left(k_{i}\right) \tilde{\Phi}_{1}(t)\left(I_{k_{i}}^{0}+\varepsilon_{1}\right) \\
& -\left(\beta_{1}+\gamma+d\right) Q_{k_{i}}^{1}(t) .
\end{aligned}
$$

Let us consider the Lyapunov function $V(t)=$ $\sum_{i=1}^{n} \frac{\theta\left(k_{i}\right) Z\left(k_{i}\right)}{\langle k\rangle} Q_{k_{i}}^{1}(t)$. We derive that

$$
\begin{aligned}
\frac{\mathrm{d} V(t)}{\mathrm{d} t}= & \sum_{i=1}^{n} \frac{\theta\left(k_{i}\right) Z\left(k_{i}\right)}{\langle k\rangle}\left[\alpha\left(k_{i}\right) \tilde{\Phi}_{1}(t)\left(I_{k_{i}}^{0}+\varepsilon_{1}\right)\right. \\
& \left.-\left(\beta_{1}+\gamma+d\right) Q_{k_{i}}^{1}(t)\right] \\
= & \tilde{\Phi}_{1}(t)\left(\beta_{1}+\mu+d\right) \\
& {\left[\sum_{i=1}^{n} \frac{p \theta\left(k_{i}\right) Z\left(k_{i}\right) \alpha\left(k_{i}\right)}{(\mu+d)\langle k\rangle\left(\beta_{1}+\gamma+d\right)}\right.} \\
& \left.+\sum_{i=1}^{n} \frac{\theta\left(k_{i}\right) Z\left(k_{i}\right) \alpha\left(k_{i}\right) \varepsilon_{1}}{\left(\beta_{1}+\mu+d\right)\langle k\rangle}-1\right] \\
= & \tilde{\Phi}_{1}(t)\left(\beta_{1}+\mu+d\right) \\
& {\left[r_{01}+\sum_{i=1}^{n} \frac{\alpha\left(k_{i}\right) \theta\left(k_{i}\right) Z\left(k_{i}\right) \varepsilon_{1}}{\left(\beta_{1}+\mu+d\right)\langle k\rangle}-1\right] . }
\end{aligned}
$$

Since $r_{01}<1$, we can choose a small enough $\varepsilon_{1}>0$ such that $r_{01}+\sum_{i=1}^{n} \frac{\alpha\left(k_{i}\right) \theta\left(k_{i}\right) Z\left(k_{i}\right) \varepsilon_{1}}{\left(\beta_{1}+\mu+d\right)\langle k\rangle}<1$. Hence, $\frac{\mathrm{d} V(t)}{\mathrm{d} t} \leq 0$ for all $Q_{k_{i}}^{1}(t) \geq 0$, and $\frac{\mathrm{d} V(t)}{\mathrm{d} t}=0$ only if $Q_{k_{i}}^{1}(t)=0$. Therefore, $Q_{k_{i}}^{1}(t)$ tends to zero as $t \rightarrow+\infty$, i.e., $\lim _{t \rightarrow \infty} Q_{k_{i}}^{1}(t)=0$. By the comparison theorem, we have $0<S_{k_{i}}^{1}(t)<Q_{k_{i}}^{1}(t)$, for all $t>0$. Therefore, $S_{k_{i}}^{1}(t)=0$ as $t \rightarrow+\infty$.

Next, we will show $S_{k_{i}}^{2}(t)$ tends to zero as $t \rightarrow \infty$. For arbitrarily enough small $\varepsilon_{2}>0$, there exists $t_{2}>0$ such that $I_{k_{i}}(t) \leq I_{k_{i}}^{0}+\varepsilon_{2}$ and $0<S_{k_{i}}^{1}(t) \leq \varepsilon_{2}$ for $t>t_{2}$. Then, for $t>t_{2}$, it has that

$$
\begin{aligned}
\frac{\mathrm{d} S_{k_{i}}^{2}(t)}{\mathrm{d} t} \leq & \rho\left(k_{i}\right) \Phi_{2}(t)\left(I_{k_{i}}^{0}+\varepsilon_{2}\right) \\
& -\left(\beta_{2}+d\right) S_{k_{i}}^{2}(t)+\gamma \varepsilon_{2} .
\end{aligned}
$$

According to the comparison system with the condition $Q_{k_{i}}^{2}(0)=S_{k_{i}}^{2}(0)>0$, let $\tilde{\Phi}_{2}(t)=\frac{\sum_{i=1}^{n} \theta\left(k_{i}\right) Z\left(k_{i}\right) Q_{k_{i}}^{2}(t)}{\langle k\rangle}$, then one has

$$
\begin{aligned}
\frac{\mathrm{d} Q_{k_{i}}^{2}(t)}{\mathrm{d} t}= & \rho\left(k_{i}\right) \tilde{\Phi}_{2}(t)\left(I_{k_{i}}^{0}+\varepsilon_{2}\right) \\
& -\left(\beta_{2}+d\right) Q_{k_{i}}^{2}(t)+\gamma \varepsilon_{2} .
\end{aligned}
$$

Let us consider the Lyapunov function $V(t)=\sum_{i=1}^{n}$ $\frac{\theta\left(k_{i}\right) Z\left(k_{i}\right)}{\langle k\rangle} Q_{k_{i}}^{2}(t)$. It has

$$
\begin{aligned}
& \frac{\mathrm{d} V(t)}{\mathrm{d} t}=\sum_{i=1}^{n} \frac{\theta\left(k_{i}\right) Z\left(k_{i}\right)}{\langle k\rangle} \\
& \quad\left[\rho\left(k_{i}\right) \tilde{\Phi}_{2}(t)\left(I_{k_{i}}^{0}+\varepsilon_{2}\right)-\left(\beta_{2}+d\right) Q_{k_{i}}^{2}(t)+\gamma \varepsilon_{2}\right] \\
& =\tilde{\Phi}_{2}(t)\left(\beta_{2}+d\right) \\
& \quad\left[\sum_{i=1}^{n} \frac{\theta\left(k_{i}\right) Z\left(k_{i}\right) \rho\left(k_{i}\right)}{\langle k\rangle\left(\beta_{2}+d\right)}\left(\frac{p}{(\mu+d)}+\varepsilon_{2}\right)\right. \\
& \left.-\frac{\gamma \varepsilon_{2}}{\tilde{\Phi}_{2}(t)\left(\beta_{2}+d\right)}-1\right]=\tilde{\Phi}_{2}(t)\left(\beta_{2}+d\right)\left[r_{02}+\sum_{i=1}^{n}\right. \\
& \left.\frac{\left(\rho\left(k_{i}\right) \theta\left(k_{i}\right) Z\left(k_{i}\right)-\frac{\gamma\langle k\rangle}{\tilde{\Phi}_{2}(t)}\right) \varepsilon_{2}}{\left(\beta_{2}+d\right)\langle k\rangle}-1\right] .
\end{aligned}
$$

Similar to the previous analysis, we can get that $\lim _{t \rightarrow \infty} S_{k_{i}}^{2}(t)=0$.

Due to $\lim _{t \rightarrow \infty} S_{k_{i}}^{1}(t)=0$ and $\lim _{t \rightarrow \infty} S_{k_{i}}^{2}(t)=0$, we can get that for arbitrarily enough small $\varepsilon_{3}>0$, there exists $t_{3}>0$ such that $0 \leq S_{k_{i}}^{1}(t) \leq \varepsilon_{3}$ and $0 \leq S_{k_{i}}^{2}(t) \leq \varepsilon_{3}$ for all $t>t_{3}$. Hence, we have

$$
\begin{aligned}
\frac{\mathrm{d} I_{k_{i}}(t)}{\mathrm{d} t} \geq & p-(\mu+d) I_{k_{i}}(t)-\left(\alpha\left(k_{i}\right)\right. \\
& \left.+\rho\left(k_{i}\right)\right) I_{k_{i}}(t) \Theta \varepsilon_{3}
\end{aligned}
$$

where $\Theta=\sum_{i=1}^{n} \frac{\theta\left(k_{i}\right) Z\left(k_{i}\right)}{\langle k\rangle}$. By using lemma 2, we have $\lim _{t \rightarrow \infty} I_{k_{i}}(t) \geq \frac{p}{(\mu+d)+\left(\alpha\left(k_{i}\right)+\rho\left(k_{i}\right)\right) \Theta \varepsilon_{3}}$. Setting $\varepsilon_{3} \rightarrow 0$, it follows that

$\lim _{t \rightarrow \infty} \inf I_{k_{i}}(t) \geq \frac{p}{d+\mu}=I_{k_{i}}^{0}$. 
From (19) and (22), it is clear that $\lim _{t \rightarrow \infty} I_{k_{i}}(t)=$ $I_{k_{i}}^{0}=\frac{p}{d+\mu}$. This proves that the equilibrium $E_{0}$ is globally asymptotically stable. The proof is completed.

Lemma 3 If $r_{01}<1$ and $r_{02}>1$, the positive solution $E^{+}$of model (1) satisfies

$$
\begin{aligned}
& \lim _{E^{+} \rightarrow E_{1}^{*}} \Phi_{1}^{+}(t)=0, \\
& \lim _{E^{+} \rightarrow E_{1}^{*}} \frac{1}{\langle k\rangle} \sum_{i=1}^{n} \rho\left(k_{i}\right) \theta\left(k_{i}\right) Z\left(k_{i}\right) I_{1 k_{i}}^{+}(t)=\beta_{2}+d .
\end{aligned}
$$

Proof Based on the definition of $\Phi_{1}(t)$, we can get that

$$
\begin{aligned}
\dot{\Phi}_{1}(t)= & \frac{\sum_{i=1}^{n} \theta\left(k_{i}\right) Z\left(k_{i}\right) \dot{S}_{k_{i}}^{1}(t)}{\langle k\rangle} \\
= & \Phi_{1}(t)\left[\frac{1}{\langle k\rangle} \sum_{i=1}^{n} \alpha\left(k_{i}\right) \theta\left(k_{i}\right) Z\left(k_{i}\right) I_{k_{i}}(t)\right. \\
& \left.-\left(\beta_{1}+\gamma+d\right)\right] \\
\leq & \Phi_{1}(t)\left[\frac{p}{\langle k\rangle(d+\mu)} \sum_{i=1}^{n} \alpha\left(k_{i}\right) \theta\left(k_{i}\right) Z\left(k_{i}\right)\right. \\
& \left.-\left(\beta_{1}+\gamma+d\right)\right] \\
= & \frac{\Phi_{1}(t)}{\beta_{1}+\gamma+d}\left(r_{01}-1\right)<0 .
\end{aligned}
$$

Then, the positive solution $E^{+}$of model (1) converges to $E_{1}^{*}$, i.e., $\lim _{E^{+} \rightarrow E_{1}^{*}} \Phi_{1}^{+}(t)=0$. From $\lim _{E^{+} \rightarrow E_{1}^{*}} \Phi_{1}(t)=0$, we obtain that $I_{1 k_{i}}^{*}=\frac{\left(d+\beta_{2}\right) S_{1 k_{i}}^{2 *}}{\rho\left(k_{i}\right) \Phi_{2}^{*}}$. Therefore,

$$
\begin{aligned}
& \lim _{E^{+} \rightarrow E_{1}^{*}} \frac{1}{\langle k\rangle} \sum_{i=1}^{n} \rho\left(k_{i}\right) \theta\left(k_{i}\right) Z\left(k_{i}\right) I_{1 k_{i}}^{+}(t) \\
& =\frac{\left(d+\beta_{2}\right)}{\Phi_{2}^{*}(t)} \frac{1}{\langle k\rangle} \sum_{i=1}^{n} \theta\left(k_{i}\right) Z\left(k_{i}\right) S_{1 k_{i}}^{2 *}(t) \\
& =d+\beta_{2} .
\end{aligned}
$$

Theorem 4 If $r_{01}<1$ and $r_{02}>1, E_{1}^{*}$ is globally asymptotically stable.
Proof We construct the Lyapunov function $V(t)$ as

$$
\begin{aligned}
V(t)= & \frac{1}{2\langle k\rangle} \sum_{i=1}^{n} \frac{1}{I_{1 k_{i}}^{*}} \theta\left(k_{i}\right) Z\left(k_{i}\right)\left(I_{k_{i}}(t)-I_{1 k_{i}}^{*}\right)^{2} \\
& +\left(\Phi_{2}(t)-\Phi_{2}^{*}-\Phi_{2}^{*} \ln \left(\frac{\Phi_{2}(t)}{\Phi_{2}^{*}}\right)\right) .
\end{aligned}
$$

The time derivative of $V(t)$ along with model (1) is given by

$$
\begin{aligned}
& \dot{V}(t)=\frac{1}{\langle k\rangle} \sum_{i=1}^{n} \frac{1}{I_{1 k_{i}}^{*}} \theta\left(k_{i}\right) Z\left(k_{i}\right)\left(I_{k_{i}}(t)-I_{1 k_{i}}^{*}\right) \dot{I}_{k_{i}}(t) \\
& +\frac{\Phi_{2}(t)-\Phi_{2}^{*}}{\Phi_{2}(t)} \dot{\Phi}_{2}(t) \\
& =\frac{1}{\langle k\rangle} \sum_{i=1}^{n} \frac{1}{I_{1 k_{i}}^{*}} \theta\left(k_{i}\right) Z\left(k_{i}\right)\left(I_{k_{i}}(t)-I_{1 k_{i}}^{*}\right) \\
& {\left[p-\alpha\left(k_{i}\right) I_{k_{i}}(t) \Phi_{1}(t)-\rho\left(k_{i}\right) I_{k_{i}}(t) \Phi_{2}(t)\right.} \\
& \left.-(d+\mu) I_{k_{i}}(t)\right]+\left(\Phi_{2}(t)-\Phi_{2}^{*}\right) \\
& {\left[\frac{1}{\langle k\rangle} \sum_{i=1}^{n} \rho\left(k_{i}\right) \theta\left(k_{i}\right) Z\left(k_{i}\right) I_{k_{i}}(t)-\left(\beta_{2}+d\right)\right]} \\
& =\frac{1}{\langle k\rangle} \sum_{i=1}^{n} \frac{1}{I_{1 k_{i}}^{*}} \theta\left(k_{i}\right) Z\left(k_{i}\right)\left(I_{k_{i}}(t)-I_{1 k_{i}}^{*}\right) \\
& {\left[\rho\left(k_{i}\right) I_{1 k_{i}}^{*} \Phi_{2}^{*}+(d+\mu) I_{1 k_{i}}^{*}\right.} \\
& \left.-\rho\left(k_{i}\right) I_{k_{i}}(t) \Phi_{2}(t)-(d+\mu) I_{k_{i}}(t)\right] \\
& +\left(\Phi_{2}(t)-\Phi_{2}^{*}\right) \\
& {\left[\frac{1}{\langle k\rangle} \sum_{i=1}^{n} \rho\left(k_{i}\right) \theta\left(k_{i}\right) Z\left(k_{i}\right) I_{k_{i}}(t)\right.} \\
& \left.-\frac{1}{\langle k\rangle} \sum_{i=1}^{n} \rho\left(k_{i}\right) \theta\left(k_{i}\right) Z\left(k_{i}\right) I_{1 k_{i}}^{*}\right] \\
& =\frac{1}{\langle k\rangle} \sum_{i=1}^{n}\left[-\frac{1}{I_{1 k_{i}}^{*}} \theta\left(k_{i}\right) Z\left(k_{i}\right)\left(d+\mu+\rho\left(k_{i}\right)\right)\right. \\
& \Phi_{2}(t)\left(I_{k_{i}}(t)-I_{1 k_{i}}^{*}\right)^{2} \\
& \left.-\theta\left(k_{i}\right) Z\left(k_{i}\right) \rho\left(k_{i}\right)\left(\Phi_{2}(t)-\Phi_{2}^{*}\right)\left(I_{k_{i}}(t)-I_{1 k_{i}}^{*}\right)\right] \\
& +\frac{1}{\langle k\rangle} \sum_{i=1}^{n} \rho\left(k_{i}\right) \theta\left(k_{i}\right) Z\left(k_{i}\right)\left(\Phi_{2}(t)\right. \\
& \left.-\Phi_{2}^{*}\right)\left(I_{k_{i}}(t)-I_{1 k_{i}}^{*}\right) \\
& =-\frac{1}{\langle k\rangle} \sum_{i=1}^{n} \frac{1}{I_{1 k_{i}}^{*}} \theta\left(k_{i}\right) Z\left(k_{i}\right)
\end{aligned}
$$




$$
\begin{aligned}
& \left(d+\mu+\rho\left(k_{i}\right) \Phi_{2}(t)\right)\left(I_{k_{i}}(t)-I_{k_{i}}^{*}\right)^{2} \\
\leq & 0 .
\end{aligned}
$$

Combining with Lemma 3, we can get that $E_{1}^{*}$ is globally asymptotically stable.

Theorem 5 If $r_{01}>1, r_{02}>1$ and $\gamma=0$, the positive equilibrium $E_{2}^{*}$ is globally asymptotically stable.

Proof We construct the Lyapunov function $V(t)$ as

$$
\begin{aligned}
V(t)= & \frac{1}{2\langle k\rangle} \sum_{i=1}^{n} \frac{1}{I_{2 k_{i}}^{*}} \theta\left(k_{i}\right) Z\left(k_{i}\right)\left(I_{k_{i}}(t)-I_{2 k_{i}}^{*}\right)^{2} \\
& +\left(\Phi_{1}(t)-\Phi_{1}^{*}-\Phi_{1}^{*} \ln \left(\frac{\Phi_{1}(t)}{\Phi_{1}^{*}}\right)\right) \\
& +\left(\Phi_{2}(t)-\Phi_{2}^{*}-\Phi_{2}^{*} \ln \left(\frac{\Phi_{2}(t)}{\Phi_{2}^{*}}\right)\right) .
\end{aligned}
$$

The time derivative of $V(t)$ along with model (1) is given by

$$
\begin{aligned}
\dot{V}(t)= & \frac{1}{\langle k\rangle} \sum_{i=1}^{n} \frac{1}{I_{2 k_{i}}^{*}} \theta\left(k_{i}\right) Z\left(k_{i}\right)\left(I_{k_{i}}(t)-I_{2 k_{i}}^{*}\right) \dot{I}_{k_{i}}(t) \\
& +\frac{\Phi_{1}(t)-\Phi_{1}^{*}}{\Phi_{1}(t)} \dot{\Phi}_{1}(t) \\
& +\frac{\Phi_{2}(t)-\Phi_{2}^{*}}{\Phi_{2}(t)} \dot{\Phi}_{2}(t) .
\end{aligned}
$$

Since

$$
\begin{aligned}
& \frac{1}{\langle k\rangle} \sum_{i=1}^{n} \frac{1}{I_{2 k_{i}}^{*}} \theta\left(k_{i}\right) Z\left(k_{i}\right)\left(I_{k_{i}}(t)-I_{2 k_{i}}^{*}\right) \dot{I}_{k_{i}}(t) \\
&=\frac{1}{\langle k\rangle} \sum_{i=1}^{n} \frac{1}{I_{2 k_{i}}^{*}} \theta\left(k_{i}\right) Z\left(k_{i}\right)\left(I_{k_{i}}(t)-I_{2 k_{i}}^{*}\right) \\
& \\
& \quad\left[p-\alpha\left(k_{i}\right) I_{k_{i}}(t) \Phi_{1}(t)-\rho\left(k_{i}\right) I_{k_{i}}(t) \Phi_{2}(t)\right. \\
&\left.\quad-(d+\mu) I_{k_{i}}(t)\right] \\
&=\frac{1}{\langle k\rangle} \sum_{i=1}^{n} \frac{1}{I_{2 k_{i}}^{*}} \theta\left(k_{i}\right) Z\left(k_{i}\right)\left(I_{k_{i}}(t)-I_{2 k_{i}}^{*}\right) \\
& {\left[\alpha\left(k_{i}\right) I_{2 k_{i}}^{*} \Phi_{1}^{*}+\rho\left(k_{i}\right) I_{2 k_{i}}^{*} \Phi_{2}^{*}+(d+\mu) I_{2 k_{i}}^{*}\right.} \\
&\left.-\alpha\left(k_{i}\right) I_{k_{i}}(t) \Phi_{1}(t)-\rho\left(k_{i}\right) I_{k_{i}}(t) \Phi_{2}(t)-(d+\mu) I_{k_{i}}(t)\right] \\
&= \frac{1}{\langle k\rangle} \sum_{i=1}^{n}\left[-\frac{1}{I_{2 k_{i}}^{*}} \theta\left(k_{i}\right) Z\left(k_{i}\right)\right. \\
& \alpha\left(k_{i}\right) \Phi_{1}(t)\left(I_{k_{i}}(t)-I_{2 k_{i}}^{*}\right)^{2} \\
&-\theta\left(k_{i}\right) Z\left(k_{i}\right) \alpha\left(k_{i}\right)\left(\Phi_{1}(t)-\Phi_{1}^{*}\right)\left(I_{k_{i}}(t)-I_{2 k_{i}}^{*}\right) \\
&-\frac{1}{I_{2 k_{i}}^{*}} \theta\left(k_{i}\right) Z\left(k_{i}\right)
\end{aligned}
$$

and

$$
\begin{aligned}
& \frac{\Phi_{1}(t)-\Phi_{1}^{*}}{\Phi_{1}(t)} \dot{\Phi}_{1}(t)+\frac{\Phi_{2}(t)-\Phi_{2}^{*}}{\Phi_{2}(t)} \dot{\Phi}_{2}(t) \\
& =\left(\Phi_{1}(t)-\Phi_{1}^{*}\right) \\
& {\left[\frac{1}{\langle k\rangle} \sum_{i=1}^{n} \alpha\left(k_{i}\right) \theta\left(k_{i}\right) Z\left(k_{i}\right) I_{k_{i}}(t)-\left(\beta_{1}+d\right)\right]} \\
& +\left(\Phi_{2}(t)-\Phi_{2}^{*}\right)\left[\frac{1}{\langle k\rangle} \sum_{i=1}^{n} \rho\left(k_{i}\right) \theta\left(k_{i}\right) Z\left(k_{i}\right)\right. \\
& \left.I_{k_{i}}(t)-\left(\beta_{2}+d\right)\right] \\
& =\left(\Phi_{1}(t)-\Phi_{1}^{*}\right) \\
& {\left[\frac{1}{\langle k\rangle} \sum_{i=1}^{n} \alpha\left(k_{i}\right) \theta\left(k_{i}\right) Z\left(k_{i}\right) I_{k_{i}}(t)-\frac{\alpha\left(k_{i}\right)}{\langle k\rangle}\right.} \\
& \left.\sum_{i=1}^{n} \theta\left(k_{i}\right) Z\left(k_{i}\right) I_{2 k_{i}}^{*}\right]+\left(\Phi_{2}(t)-\Phi_{2}^{*}\right) \\
& {\left[\frac{1}{\langle k\rangle} \sum_{i=1}^{n} \rho\left(k_{i}\right) \theta\left(k_{i}\right) Z\left(k_{i}\right) I_{k_{i}}(t)\right.} \\
& \left.-\frac{\rho\left(k_{i}\right)}{\langle k\rangle} \sum_{i=1}^{n} \theta\left(k_{i}\right) Z\left(k_{i}\right) I_{2 k_{i}}^{*}\right] \\
& =\left(\Phi_{1}(t)-\Phi_{1}^{*}\right)\left[\frac{1}{\langle k\rangle} \sum_{i=1}^{n} \alpha\left(k_{i}\right) \theta\left(k_{i}\right) Z\left(k_{i}\right)\right. \\
& \left.\left(I_{k_{i}}(t)-I_{2 k_{i}}^{*}\right)\right]+\left(\Phi_{2}(t)-\Phi_{2}^{*}\right) \\
& {\left[\frac{1}{\langle k\rangle} \sum_{i=1}^{n} \rho\left(k_{i}\right) \theta\left(k_{i}\right) Z\left(k_{i}\right) \quad\left(I_{k_{i}}(t)-I_{2 k_{i}}^{*}\right)\right] \text {. }}
\end{aligned}
$$

Then, combining with (30) and (31), one has

$$
\begin{array}{r}
\dot{V}(t)=-\frac{1}{\langle k\rangle} \sum_{i=1}^{n}\left[\frac{1}{I_{2 k_{i}}^{*}} \theta\left(k_{i}\right) Z\left(k_{i}\right)\right. \\
\left.\left.\alpha\left(k_{i}\right) \Phi_{1}(t)\right)\left(I_{k_{i}}(t)-I_{2 k_{i}}^{*}\right)^{2}\right]
\end{array}
$$




$$
\begin{aligned}
& -\frac{1}{\langle k\rangle} \sum_{i=1}^{n}\left[\frac{1}{I_{2 k_{i}}^{*}} \theta\left(k_{i}\right) Z\left(k_{i}\right)\left(d+\mu+\rho\left(k_{i}\right)\right)\right. \\
& \left.\left.\Phi_{2}(t)\right)\left(I_{k_{i}}(t)-I_{2 k_{i}}^{*}\right)^{2}\right] \\
& \leq 0
\end{aligned}
$$

Thus, $E_{2}^{*}$ is globally asymptotically stable.

\section{Continuous control}

When the rumor persists and no measures are taken to limit its spreading, it will have a certain negative impact on social security and stability. Therefore, how to control the spread of rumor and protect the society from huge losses is a problem that the government should consider. Maybe educating the spreaders is a feasible strategy to control the spread of rumor. Hence, the educational mechanism is considered to the rumor spreaders in model (1). The specific model is as follows:

$$
\left\{\begin{aligned}
\frac{\mathrm{d} I_{k_{i}}(t)}{\mathrm{d} t}= & p-\alpha\left(k_{i}\right) I_{k_{i}}(t) \Phi_{1}(t) \\
& -\rho\left(k_{i}\right) I_{k_{i}}(t) \Phi_{2}(t)-(\mu+d) I_{k_{i}}(t) \\
\frac{\mathrm{d} S_{k_{i}}^{1}(t)}{\mathrm{d} t}= & \alpha\left(k_{i}\right) I_{k_{i}}(t) \Phi_{1}(t)-\beta_{1} S_{k_{i}}^{1}(t) \\
& -(\gamma+d) S_{k_{i}}^{1}(t)-\tau S_{k_{i}}^{1}(t) \\
\frac{\mathrm{d} S_{k_{i}}^{2}(t)}{\mathrm{d} t}= & \rho\left(k_{i}\right) I_{k_{i}}(t) \Phi_{2}(t)-\beta_{2} S_{k_{i}}^{2}(t) \\
& +\gamma S_{k_{i}}^{1}(t)-d S_{k_{i}}^{2}(t)-\tau S_{k_{i}}^{2}(t) \\
\frac{\mathrm{d} R_{k_{i}}(t)}{\mathrm{d} t}= & \beta_{1} S_{k_{i}}^{1}(t) \\
& +\beta_{2} S_{k_{i}}^{2}(t)+\mu I_{k_{i}}(t)-d R_{k_{i}}(t)
\end{aligned}\right.
$$

where $0<\tau<1$ represents the educational influence on rumor spreaders. The purpose of our control on the spreaders is to stop the propagation of rumor. Hence, we only need to prove that the spreaders eventually tend to be extinct under the control. How much the control influence can make the rumor extinct is the issue that we need to further study next. Here, the following theorem is given to help us solve this issue.

Theorem 6 For model (33), the rumor can die out if the following condition is satisfied

$$
\begin{aligned}
\tau & >\max \left\{\frac{p \sum_{i=1}^{n} \alpha\left(k_{i}\right) \theta\left(k_{i}\right) Z\left(k_{i}\right)}{\langle k\rangle(d+\mu)}\right. \\
& -\left(\beta_{1}+d+\gamma\right), \frac{p \sum_{i=1}^{n} \rho\left(k_{i}\right) \theta\left(k_{i}\right) Z\left(k_{i}\right)}{\langle k\rangle(d+\mu)} \\
& \left.-\left(\beta_{2}+d\right)\right\} .
\end{aligned}
$$

Proof For model (33), similar to the analysis of Theorem 1 , the basic reproduction number $r_{0}^{*}$ is

$$
\begin{aligned}
r_{0}^{*}= & \max \left\{\frac{p \sum_{i=1}^{n} \alpha\left(k_{i}\right) \theta\left(k_{i}\right) Z\left(k_{i}\right)}{\langle k\rangle(d+\mu)\left(\beta_{1}+\gamma+d+\tau\right)},\right. \\
& \left.\frac{p \sum_{i=1}^{n} \rho\left(k_{i}\right) \theta\left(k_{i}\right) Z\left(k_{i}\right)}{\langle k\rangle(d+\mu)\left(\beta_{2}+d+\tau\right)}\right\} .
\end{aligned}
$$

As we know that $r_{0}^{*}$ is the condition to decide whether the rumor spreads or is extinct, if $r_{0}^{*}<1$, the rumor will die out. Therefore, we get

$$
\begin{aligned}
\tau> & \max \left\{\frac{p \sum_{i=1}^{n} \alpha\left(k_{i}\right) \theta\left(k_{i}\right) Z\left(k_{i}\right)}{\langle k\rangle(d+\mu)}-\left(\beta_{1}+d+\gamma\right),\right. \\
& \left.\frac{p \sum_{i=1}^{n} \rho\left(k_{i}\right) \theta\left(k_{i}\right) Z\left(k_{i}\right)}{\langle k\rangle(d+\mu)}-\left(\beta_{2}+d\right)\right\} .
\end{aligned}
$$

The rumor can be effectively restrained when the control intensity of the propagator satisfies model (35), which can be seen in the later numerical simulations.

\section{Event-triggered impulsive control}

We can use continuous control mechanism to suppress the spreading of the rumor and make it die out quickly and effectively. In fact, it is challenging and sometimes impossible to control the rumor spreaders continuously. Therefore, the impulsive control can effectively overcome this deficiency. To save limited resources, we propose an event-triggered impulsive control strategy, which only needs to control the rumor spreaders at some discrete instants. In the following, we give the main analysis process.

To introduce the event-triggered impulsive control, we let $S^{1}(t)=\left(S_{k_{1}}^{1}(t), S_{k_{2}}^{1}(t), \ldots, S_{k_{n}}^{1}(t)\right)^{T}$, $S^{2}(t)=\left(S_{k_{1}}^{2}(t), S_{k_{2}}^{2}(t), \ldots, S_{k_{n}}^{2}(t)\right)^{T}$ and $S(t)=$ $\left(S^{1}(t), S^{2}(t)\right)^{T}$. Then, the event-triggered impulsive control is given by 
$\begin{cases}X(t)=-q S(t) & t \in\left[t_{k-1}, t_{k}\right) \\ \Delta S\left(t_{k}\right)=\epsilon X\left(t_{k}\right), & t=t_{k}\end{cases}$

where $\Delta S\left(t_{k}\right)=S\left(t_{k}^{+}\right)-S\left(t_{k}^{-}\right), S\left(t_{k}\right)=S\left(t_{k}^{+}\right), \epsilon$ is an impulsive strength. The impulsive sequence $t_{k}$ satisfies $0=t_{0}<t_{1}<\cdots<t_{k}<, \ldots, \lim _{k \rightarrow \infty} t_{k}=\infty$, and $0<q<1$ represents the control effect.

Under the impulsive control (36), we present the following control system.

When $t \in\left[t_{k-1}, t_{k}\right)$ :

$$
\left\{\begin{aligned}
\frac{\mathrm{d} I_{k_{i}}(t)}{\mathrm{d} t}= & p-\alpha\left(k_{i}\right) I_{k_{i}}(t) \Phi_{1}(t) \\
& -\rho\left(k_{i}\right) I_{k_{i}}(t) \Phi_{2}(t)-(\mu+d) I_{k_{i}}(t) \\
\frac{\mathrm{d} S_{k_{i}}^{1}(t)}{\mathrm{d} t}= & \alpha\left(k_{i}\right) I_{k_{i}}(t) \Phi_{1}(t) \\
& -\beta_{1} S_{k_{i}}^{1}(t)-(\gamma+d) S_{k_{i}}^{1}(t) \\
\frac{\mathrm{d} S_{k_{i}}^{2}(t)}{\mathrm{d} t}= & \rho\left(k_{i}\right) I_{k_{i}}(t) \Phi_{2}(t) \\
& -\beta_{2} S_{k_{i}}^{2}(t)+\gamma S_{k_{i}}^{1}(t)-d S_{k_{i}}^{2}(t) \\
\frac{\mathrm{d} R_{k_{i}}(t)}{\mathrm{d} t}= & \beta_{1} S_{k_{i}}^{1}(t)+\beta_{2} S_{k_{i}}^{2}(t) \\
& +\mu I_{k_{i}}(t)-d R_{k_{i}}(t) .
\end{aligned}\right.
$$

When $t=t_{k}$ :

$$
\left\{\begin{array}{l}
I_{k_{i}}\left(t_{k}^{+}\right)=I_{k_{i}}\left(t_{k}\right) \\
S_{k_{i}}^{1}\left(t_{k}^{+}\right)=(1-\epsilon q) S_{k_{i}}^{1}\left(t_{k}\right) \\
S_{k_{i}}^{2}\left(t_{k}^{+}\right)=(1-\epsilon q) S_{k_{i}}^{2}\left(t_{k}\right) \\
R_{k_{i}}\left(t_{k}^{+}\right)=\epsilon q\left(S_{k_{i}}^{1}\left(t_{k}\right)+S_{k_{i}}^{2}\left(t_{k}\right)\right)+R_{k_{i}}\left(t_{k}\right) .
\end{array}\right.
$$

Let $t_{k}$ be the triggering time sequence of rumor propagation variable, and $t_{k+1}=\inf \left\{t \mid g(t) \geq 0, t>t_{k}\right\}$. The event-trigger function is defined as

$g(t)=\|e(t)\|^{2}-\frac{\zeta}{2}\left\|X\left(t_{k}\right)\right\|^{2}-\frac{\sigma}{\left(t-t_{0}\right)^{2}}$

where $\varsigma>0, \sigma>0$ and $e(t)=X\left(t_{k}\right)-X(t)$.

Remark 5 Different from existing time-dependent impulsive control, we apply the event-triggered impulsive control to suppress the spreading of rumors in this paper. The impulsive instants depend on the eventtrigger function (39). If $g(t) \geq 0$, the impulsive control (36) occurs. Then, $e(t)=0$ and we get that the eventtrigger function $g(t)<0$ from (39). Over time, the sample error $\|e(t)\|$ will increase. The next trigger will occur when it satisfies $g(t) \geq 0$. The term $\frac{\sigma}{\left(t-t_{0}\right)^{2}}$ is used to ensure that the Zeno behavior will not happen.

Let $\xi\left(k_{i}\right)=\theta\left(k_{i}\right) Z\left(k_{i}\right)$, and $\xi=\left(\xi\left(k_{1}\right), \xi\left(k_{2}\right), \ldots\right.$, $\left.\xi\left(k_{n}\right)\right)^{T}$ for $i=1,2, \ldots, n$. Then, for any $t \in$ $\left[t_{k}, t_{k+1}\right), k \in N$, the derivatives of $S_{k_{i}}^{1}(t)$ and $S_{k_{i}}^{2}(t)$ in (37) are converted into

$$
\begin{aligned}
\frac{\mathrm{d} S^{1}(t)}{\mathrm{d} t} & =\left[\frac{1}{\langle k\rangle} \Xi_{1}(t)-\left(\beta_{1}+\gamma+d\right) \mathcal{I}_{n}\right] S^{1}(t) \\
\frac{\mathrm{d} S^{2}(t)}{\mathrm{d} t} & =\left[\frac{1}{\langle k\rangle} \Xi_{2}(t)-\left(\beta_{2}+d\right) \mathcal{I}_{n}\right] S^{2}(t)+\gamma S^{1}(t)
\end{aligned}
$$

where $\Xi_{1}(t)=\left(\begin{array}{c}\alpha\left(k_{1}\right) I_{k_{1}}(t) \\ \alpha\left(k_{2}\right) I_{k_{2}}(t) \\ \vdots \\ \alpha\left(k_{n}\right) I_{k_{n}}(t)\end{array}\right) \xi^{T}$ and $\Xi_{2}(t)=$ $\left(\begin{array}{c}\rho\left(k_{1}\right) I_{k_{1}}(t) \\ \rho\left(k_{2}\right) I_{k_{2}}(t) \\ \vdots \\ \rho\left(k_{n}\right) I_{k_{n}}(t)\end{array}\right) \xi^{T}$

Due to $I_{k_{i}}(t) \leq \frac{p}{d+\mu}$, we replace $I_{k_{i}}(t)$ in $\Xi_{1}(t)$ and $\Xi_{2}(t)$ with $\frac{p}{d+\mu}$, then the new matrices are denoted by $\bar{\Xi}_{1}$ and $\bar{\Xi}_{2}$, respectively. We can get the following equation

$$
\begin{aligned}
\frac{\mathrm{d} S(t)}{\mathrm{d} t} & =\left(\begin{array}{cc}
\frac{1}{\langle k\rangle} \bar{\Xi}_{1}-\left(\beta_{1}+\gamma+d\right) \mathcal{I}_{n} & 0 \\
\gamma \mathcal{I}_{n} & \frac{1}{\langle k\rangle} \bar{\Xi}_{2}-\left(\beta_{2}+d\right) \mathcal{I}_{n}
\end{array}\right) S(t) \\
& =\bar{\omega} S(t)
\end{aligned}
$$

where $\bar{\omega}=\left(\begin{array}{cc}\frac{1}{\langle k\rangle} \bar{\Xi}_{1}-\left(\beta_{1}+\gamma+d\right) \mathcal{I}_{n} & 0 \\ \gamma \mathcal{I}_{n} & \frac{1}{\langle k\rangle} \bar{\Xi}_{2}-\left(\beta_{2}+d\right) \mathcal{I}_{n}\end{array}\right)$.

From the principle of comparison, if the origin of Eq. (41) is globally asymptotically stable, the origin of Eq. (40) is also globally asymptotically stable. Therefore, in the following, we will prove the origin of Eq. (41) is globally asymptotically stable under control (36).

Before giving the main results, the following useful lemma is presented.

Lemma 4 [32] Let $\mathbf{w}(t)$ be nonnegative and continuous function on $\left[t_{0},+\infty\right)$. If the following inequality is satisfied

$\mathbf{w}(t) \leq Y+\int_{t_{0}}^{t} \varrho \mathbf{w}(s) \mathrm{d} s$ 
where $Y$ is a nonnegative constant, and $\varrho$ is a constant, then

$\mathbf{w}(t) \leq Y \exp \{\varrho \mathbf{w}(s)\}, \quad t \in\left[t_{0},+\infty\right)$.

Theorem 7 Consider model (1) under the impulsive event-triggered control (36) with the event-trigger function (39). If there exist positive numbers $\varsigma>0$, $\sigma>0,0<\epsilon<1,0<q<1$ and a positive definite matrix $U$ such that

$$
\begin{aligned}
& \lim _{t \rightarrow \infty}\left[k \ln (1-\epsilon q)+\frac{k+1}{2} \ln \left(\frac{\lambda_{\max }(U)}{\lambda_{\min }(U)}\right)\right. \\
& \left.+\frac{\lambda_{\max }(\phi)}{2 \lambda_{\min }(U)}\left(t-t_{0}\right)\right] \rightarrow-\infty
\end{aligned}
$$

where $\lambda_{\max }(U)$ and $\lambda_{\min }(U)$ represent the maximal and minimal eigenvalues of $U$, respectively. $\lambda_{\max }(\phi)$ represents the maximum eigenvalue of $\phi$, and $\phi=$ $\bar{\omega} U+U^{T} \bar{\omega}$. Then, the rumor will fade away. Moreover, the Zeno behavior does not exist.

Proof For any $t \in\left[t_{k}, t_{k+1}\right), k \in N_{+}$, we construct the following Lyapunov function

$$
V(t)=S^{T}(t) U S(t)
$$

where $U$ is a positive definite matrix.

The time derivative of $V(t)$ is

$$
\begin{aligned}
\dot{V}(t) & =S^{T}(t) U \dot{S}(t)+\dot{S}^{T}(t) U S(t) \\
& \leq S^{T}(t)\left(\bar{\omega}^{T} U+U \bar{\omega}\right) S(t) \\
& \leq \lambda_{\max }(\phi) S^{T}(t) S(t)
\end{aligned}
$$

where $\lambda_{\max }(\phi)$ is the largest eigenvalue of the matrix $\phi=\bar{\omega}^{T} U+U \bar{\omega}$.

For $t \in\left[t_{0}, t_{1}\right)$,

$$
\begin{aligned}
\lambda_{\min }(U)\|S(t)\|^{2} \leq & V(t) \leq V\left(t_{0}\right) \\
& +\int_{t_{0}}^{t} \lambda_{\max }(\phi)\|S(s)\|^{2} \mathrm{~d} s \\
\leq & \lambda_{\max }(U)\left\|S\left(t_{0}\right)\right\|^{2} \\
& +\int_{t_{0}}^{t} \lambda_{\max }(\phi)\|S(s)\|^{2} \mathrm{~d} s .
\end{aligned}
$$

Based on Gronwall's inequality, we have

$$
\begin{aligned}
\|S(t)\|^{2} & \leq \frac{\lambda_{\max }(U)}{\lambda_{\min }(U)}\left\|S\left(t_{0}\right)\right\|^{2}+\int_{t_{0}}^{t} \frac{\lambda_{\max }(\phi)}{\lambda_{\min }(U)}\|S(s)\|^{2} \mathrm{~d} s \\
& \leq \frac{\lambda_{\max }(U)}{\lambda_{\min }(U)}\left\|S\left(t_{0}\right)\right\|^{2} \exp \left\{\frac{\lambda_{\max }(\phi)}{\lambda_{\min }(U)}\left(t-t_{0}\right)\right\} .
\end{aligned}
$$

For the case of $t=t_{1}$, we have

$$
\begin{aligned}
\left\|S\left(t_{1}\right)\right\|^{2} & \leq(1-\epsilon q)^{2} \frac{\lambda_{\max }(U)}{\lambda_{\min }(U)}\left\|S\left(t_{0}\right)\right\|^{2} \\
& \exp \left\{\frac{\lambda_{\max }(\phi)}{\lambda_{\min }(U)}\left(t_{1}-t_{0}\right)\right\} .
\end{aligned}
$$

For $t \in\left[t_{1}, t_{2}\right)$, it has

$$
\begin{aligned}
\|S(t)\|^{2} \leq & \frac{\lambda_{\max }(U)}{\lambda_{\min }(U)}\left\|S\left(t_{1}\right)\right\|^{2} \\
& \exp \left\{\frac{\lambda_{\max }(\phi)}{\lambda_{\min }(U)}\left(t-t_{1}\right)\right\} \\
\leq & (1-\epsilon q)^{2}\left(\frac{\lambda \max (U)}{\lambda_{\min }(U)}\right)^{2}\left\|S\left(t_{1}\right)\right\|^{2} \\
& \exp \left\{\frac{\lambda_{\max }(\phi)}{\lambda_{\min }(U)}\left(t-t_{0}\right)\right\} .
\end{aligned}
$$

For the case of $t=t_{2}$, we have

$$
\begin{aligned}
\left\|S\left(t_{2}\right)\right\|^{2} & \leq(1-\epsilon q)^{4}\left(\frac{\lambda_{\max }(U)}{\lambda_{\min }(U)}\right)^{2}\left\|S\left(t_{0}\right)\right\|^{2} \\
& \exp \left\{\frac{\lambda_{\max }(\phi)}{\lambda_{\min }(U)}\left(t_{2}-t_{0}\right)\right\} .
\end{aligned}
$$

Thus, for any $t \in\left[t_{k}, t_{k+1}\right)$, one can obtain that

$$
\begin{aligned}
\|S(t)\|^{2} & \leq(1-\epsilon q)^{2 k}\left(\frac{\lambda_{\max }(U)}{\lambda_{\min }(U)}\right)^{k+1}\left\|S\left(t_{0}\right)\right\|^{2} \\
& \exp \left\{\frac{\lambda_{\max }(\phi)}{\lambda_{\min }(U)}\left(t-t_{0}\right)\right\} .
\end{aligned}
$$

By using condition (44), one has $\lim _{t \rightarrow \infty}\|S(t)\|=0$. Then, the rumor dies out.

Remark 6 In Theorem 7, the condition of rumor extinction (44) is given. In fact, it can be satisfied by properly choosing control strength $\epsilon$ and $q$. When the eventtriggered interval is bounded, there exist constants $T_{\max }$ and $T_{\min }$ such that $T_{\min } \leq t_{k+1}-t_{k} \leq T_{\max }$, $k \in N$. For any event-triggered instant $t_{k}$, it has $\frac{t}{T_{\max }} \leq k \leq \frac{t}{T_{\min }}$. If the parameters $\epsilon$ and $q$ satisfy 
$\frac{\ln (1-\epsilon q)}{T_{\max }}+\frac{1}{2 T_{\min }} \ln \left(\frac{\lambda_{\max }(U)}{\lambda_{\min }(U)}+\frac{\lambda_{\max }(\phi)}{2 \lambda_{\min }(U)}\right)<0$, then condition (44) can be satisfied.

In the next, we will prove that there is no Zeno behavior under the event-triggered impulsive control strategy. Let $\dot{S}(t)=\bar{F} S(t), \bar{F}=\left(\bar{f}_{1}^{T}, \bar{f}_{2}^{T}, \ldots, \bar{f}_{2 n}^{T}\right)^{T}$, then $\dot{S}_{i}(t)=\bar{f}_{i} S(t), \bar{f}_{i}=\left(\bar{f}_{i 1}, \bar{f}_{i 2}, \cdots, \bar{f}_{i, 2 n}\right), i=$ $1,2, \ldots, 2 n$.

For $i=1,2, \ldots, n$,

$\bar{f}_{i j}=\left\{\begin{array}{l}\frac{p \alpha\left(k_{i j}\right) \xi\left(k_{j}\right)}{\langle k\rangle(d+\mu)}-\left(\beta_{1}+\gamma+d\right), \quad i=j, \\ \frac{p \alpha\left(k_{i j}\right) \xi\left(k_{j}\right)}{\langle k\rangle(d+\mu)}, \quad i \neq j, j=1,2, \ldots, n, \\ 0, \quad i \neq j, j=n+1, \ldots, 2 n .\end{array}\right.$

For $i=n+1, \ldots, 2 n$,

$\bar{f}_{i j}=\left\{\begin{array}{l}\frac{p \rho\left(k_{i}\right) \xi\left(k_{j}\right)}{\langle k\rangle(d+\mu)}-\left(\beta_{2}+d\right), \quad i=j, \\ \frac{p \rho\left(k_{i}\right) \xi\left(k_{j}\right)}{\langle k\rangle(d+\mu)}, \quad i \neq j, j=n+1, \ldots, 2 n, \\ \gamma, \quad j=i-n, \\ 0, \quad \text { otherwise. }\end{array}\right.$

For any $t \in\left[t_{k}, t_{k+1}\right)$, suppose that $\Delta(t)=$ $e^{T}(t) e(t)$. We have

$\dot{\Delta}(t)=\dot{e}^{T}(t) e(t)+e^{T}(t) \dot{e}(t)$.

Due to $e(t)=X\left(t_{k}\right)-X(t)$ and $\dot{e}(t)=-\dot{X}(t)=$ $q \bar{F} S(t)$. It has

$$
\begin{aligned}
\dot{\Delta}(t) & =2 q e^{T}(t) \bar{F} S(t) \\
& \leq e^{T}(t) e(t)+\|\bar{F}\|^{2} X^{T}(t) X(t) \\
& \leq e^{T}(t) e(t)+2\|\bar{F}\|^{2}\left(e^{T}(t) e(t)+X^{T}\left(t_{k}\right) X\left(t_{k}\right)\right) \\
& \leq\left(1+2\|F\|^{2}\right) \Delta(t)+2\|\bar{F}\|^{2}\left\|X\left(t_{k}\right)\right\|^{2}
\end{aligned}
$$

It yields that

$$
\begin{aligned}
\|e(t)\|^{2} \leq & \frac{2\|\bar{F}\|^{2}\left\|X\left(t_{k}\right)\right\|^{2}}{1+2\|\bar{F}\|^{2}} \\
& \left(\exp \left\{\left(1+2\|\bar{F}\|^{2}\right)\left(t-t_{k}\right)\right\}-1\right) .
\end{aligned}
$$

The next event will not be triggered until trigger function (39) crosses zero, i.e.,

$$
\begin{aligned}
\|e(t)\|^{2} & =\frac{\varsigma}{2}\left\|X\left(t_{k}\right)\right\|^{2}+\frac{\sigma}{\left(t-t_{0}\right)^{2}} \\
& \leq \frac{2\|\bar{F}\|^{2}\left\|X\left(t_{k}\right)\right\|^{2}}{1+2\|\bar{F}\|^{2}} \\
& \left(\exp \left\{\left(1+2\|\bar{F}\|^{2}\right)\left(t-t_{k}\right)\right\}-1\right) .
\end{aligned}
$$

Therefore, for any $t \in\left[t_{k}, t_{k+1}\right)$,

$$
\begin{aligned}
\frac{\sigma}{\left(t-t_{0}\right)^{2}} \leq & \frac{2\|\bar{F}\|^{2}\left\|X\left(t_{k}\right)\right\|^{2}}{1+2\|\bar{F}\|^{2}} \\
& \left(\exp \left\{\left(1+2\|\bar{F}\|^{2}\right)\left(t-t_{k}\right)\right\}-1\right) .
\end{aligned}
$$

Denote $T_{k}=t_{k+1}-t_{k}$ and $t=t_{k+1}$, one can obtain

$$
\begin{aligned}
\frac{\sigma}{\left(T_{k}+t_{k}-t_{0}\right)^{2}} \leq & \frac{2\|\bar{F}\|^{2}\left\|X\left(t_{k}\right)\right\|^{2}}{1+2\|\bar{F}\|^{2}} \\
& \left(\exp \left\{\left(1+2\|\bar{F}\|^{2}\right) T_{k}\right\}-1\right) .
\end{aligned}
$$

If $T_{k}=0$, then $\frac{\sigma}{\left(t_{k}-t_{0}\right)^{2}} \leq 0$. This is not true. Therefore, $T_{k}>0$ for any $k$, i.e., the Zeno behavior is excluded.

Remark 7 In this paper, the event-triggered impulsive control strategy is used to restrain the spread of rumor in a multilingual environment. For the sake of convenience, we control the spreaders in each layer simultaneously. It is found that as long as the control intensity meets some certain conditions, the rumor spreaders will die out eventually. However, when the network is tremendous, it is laborious to implement the centralized event-triggered control strategy. Consequently, in the future work, we will further study the distributed eventtriggered control strategy. Maybe the event-triggered condition only depends on the number of propagations in each layer of network.

\section{Numerical example}

In this section, the dynamic characteristic of the proposed rumor propagation model is analyzed by numerical simulation.

This paper considers a rumor propagation model with two languages under heterogeneous networks. In the simulation, we choose the degree of network as $k_{i}$ for $i=1, \ldots, 50$. Suppose that the network obeys power law distribution, and we choose $Z\left(k_{i}\right)=\frac{k_{i}^{-2.5}}{1.74}$; 
from $\sum_{i=1}^{n} Z\left(k_{i}\right)=1$, we can figure out that the average degree $\langle k\rangle=1.74$. Because people with different degrees may have diverse infection rates, the infection rates are always bounded. Hence, the infectivity is designed as $\theta\left(k_{i}\right)=\frac{k_{i}^{0.5}}{1+k_{i}^{0.5}}$. The initial value of model

(1) is selected as follows: $I_{k_{i}}(0)=0.7+\frac{k}{400}, S_{k_{i}}^{1}(0)=$ $0.1-\frac{k}{800}, S_{k_{i}}^{2}(0)=0.15-\frac{k}{1600}, R_{k_{i}}(0)=0.05-\frac{k}{1600}$.

\subsection{Stability of rumor-free equilibrium point}

Case 1: In model (1), we choose $\alpha\left(k_{i}\right)=0.6, \rho\left(k_{i}\right)=$ $0.38, \gamma=0.08, \beta_{1}=0.05, \beta_{2}=0.08, p=0.01$, $d=0.01$ and $\mu=0.005$. By simple calculation, it is derived that the basic reproduction $r_{0} \approx 0.88<1$. Based on Theorem 1, model (1) has a rumor-free equilibrium $E_{0}$. From Theorem $3, E_{0}$ is globally asymptotically stable, which is verified in Fig. 2 with $k_{50}=50$. Through the simulation, it can be seen that the number of rumor spreaders increases firstly, then it decreases, and it dies out finally. When the rumor spreaders disappear, $R\left(k_{i}\right)=1-\frac{p}{d+\mu}=\frac{1}{3}$. This means that one-third of the people on the network have received the rumor eventually. Moreover, we also simulate the rumor propagation model with different initial values when $k_{i} \in[1,50]$. As shown in Fig. 3, for individuals with different degrees, the system has the same equilibrium point. Finally, we choose $\alpha\left(k_{i}\right)=0.5+0.002 k$, $\rho\left(k_{i}\right)=0.28+0.002 k, \gamma=0.08, \beta_{1}=0.05$, $\beta_{2}=0.08, p=0.01, d=0.01, \mu=0.005$. By a simple calculation, we get $r_{0}<1$. As shown in Fig. 4, the rumor-free equilibrium point is also $E_{0}$. This means that different infection rates only affect the basic reproduction number number $r_{0}$, but do not affect the value of rumor-free equilibrium point.

\subsection{Stability of rumor equilibrium point}

Case 2: We choose $\alpha\left(k_{i}\right)=0.5, \rho\left(k_{i}\right)=0.2, \gamma=$ $0.08, \beta_{1}=0.015, \beta_{2}=0.008, p=0.01, d=0.01$, $\mu=0.005$. By simple calculation, it is derived that $r_{01} \approx 0.92<1$ and $r_{02} \approx 2.27>1$. Model (1) has an equilibrium point $E_{1}^{*}$. From Theorem $4, E_{1}^{*}$ is globally asymptotically stable, which is verified in Fig. 5 with $k_{i}=1$. Through the simulation, it can be seen that the rumor Spreaders 2 will continuously exist as time goes by.

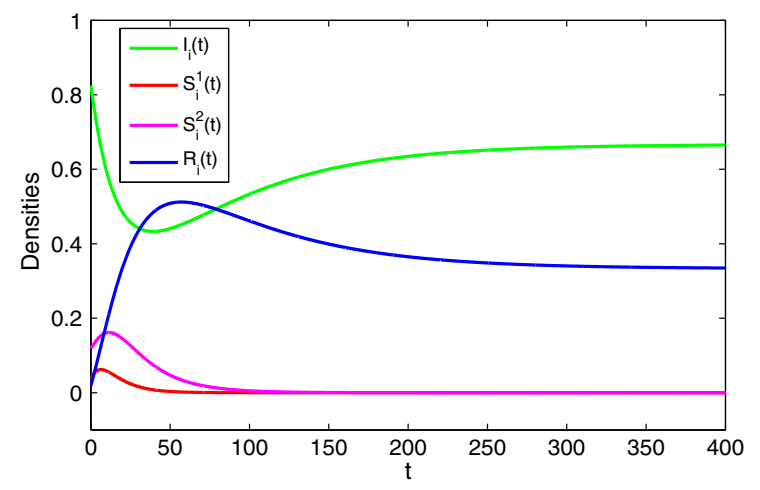

Fig. 2 Dynamics of model (1) with $r_{0}<1$ and $k_{50}=50$

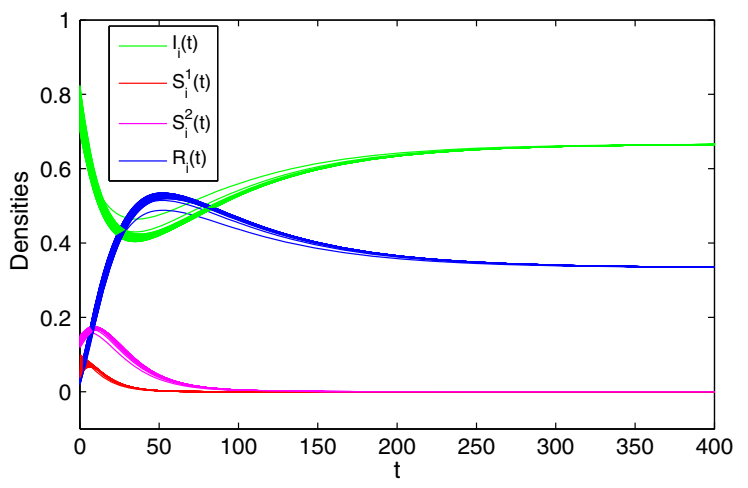

Fig. 3 Dynamics of model (1) with $r_{0}<1$ and $k_{i} \in[1,50]$

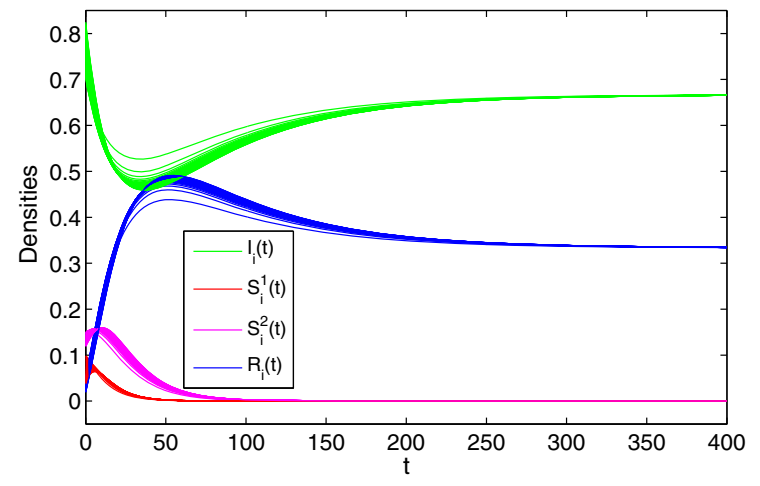

Fig. 4 Dynamics of model (1) with $r_{0}<1$ and different $\alpha\left(k_{i}\right)$, $\beta\left(k_{i}\right)$

In addition, we also simulate the rumor propagation model with different infection rates. When we choose $\alpha\left(k_{i}\right)=0.5-0.001 k, \rho\left(k_{i}\right)=0.2+0.002 k, \gamma=0.08$, $\beta_{1}=0.015, \beta_{2}=0.008, p=0.01, d=0.01$, $\mu=0.005$, By a simple calculation, we get $r_{01}<1$ and $r_{02}>1$. The simulation is shown in Fig. 6, in which 


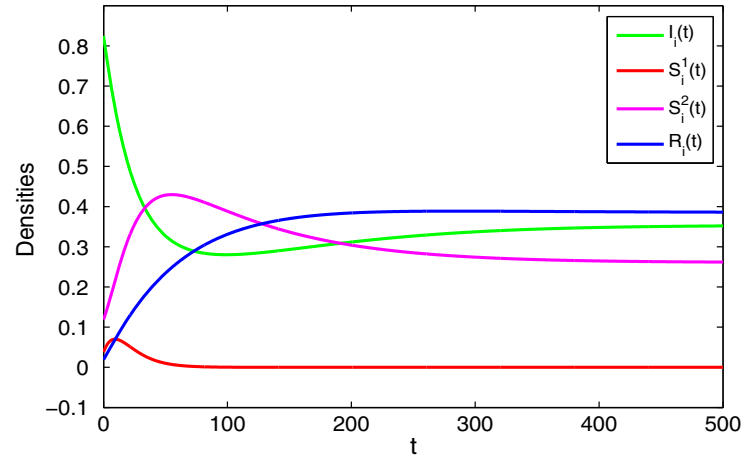

Fig. 5 Dynamics of model (1) with $r_{01}<1, r_{02}>1$ and $k_{50}=$ 50

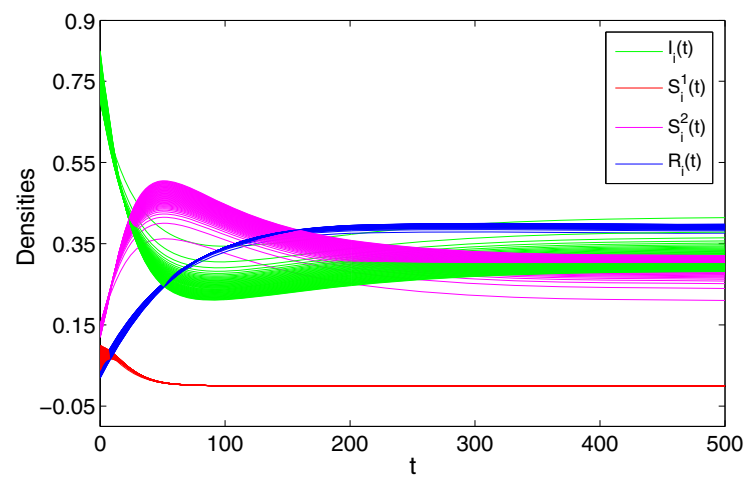

Fig. 6 Dynamics of model (1) with $r_{01}<1, r_{02}>1$ and different $\alpha\left(k_{i}\right), \beta\left(k_{i}\right)$

the rumor equilibrium point of $E_{1}^{*}$ depends on the individual degree of the network. That means the infection rate not only affects the basic reproduction number $r_{0}$, but also affects the value of rumor equilibrium point.

Case 3: We choose $\alpha\left(k_{i}\right)=0.5, \rho\left(k_{i}\right)=0.475, \gamma=0$, $\beta_{1}=0.0056, \beta_{2}=0.005, p=0.01, d=0.01$, $\mu=0.005$. By simple calculation, it is easily obtained that $r_{01} \approx 6.56>1, r_{02} \approx 6.47>1$. According to Theorem 1 and Theorem 5, model (1) has a positive equilibrium $E_{2}^{*}$, which is globally asymptotically stable. Figure 7 is given to verify the correctness of the theorems. Through the simulation, it can be seen that rumor spreaders $S^{1}\left(k_{i}\right)$ and $S^{2}\left(k_{i}\right)$ eventually tend to two constants as time goes on. Moreover, we also simulate the rumor propagation model with different infection rates. Choosing $\alpha\left(k_{i}\right)=0.4+0.002 k$, $\rho\left(k_{i}\right)=0.375+0.002 k, \gamma=0, \beta_{1}=0.0056$, $\beta_{2}=0.005, p=0.01, d=0.01, \mu=0.005$, it is easy to get $r_{01}>1$ and $r_{02}>1$. The simulation result

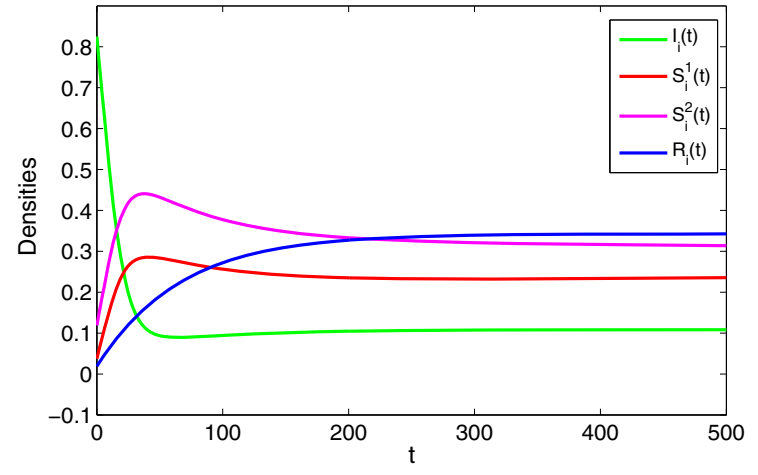

Fig. 7 Dynamics of model (1) with $r_{01}>1, r_{02}>1$ and $k_{50}=$ 50

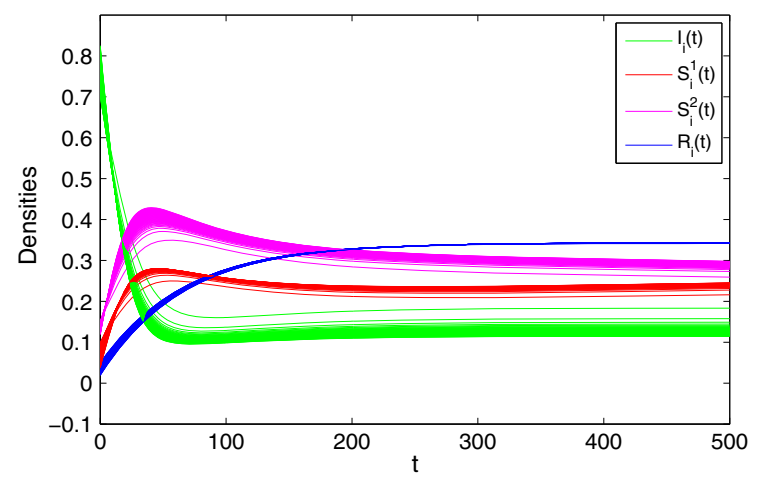

Fig. 8 Dynamics of model (1) with $r_{01}>1, r_{02}>1$ and different $\alpha\left(k_{i}\right), \beta\left(k_{i}\right)$

is shown in Fig. 8. This means that different infection rates both affect the basic reproduction number $r_{0}$ and affect the value of rumor equilibrium point. Based on Theorem 1, one can also find that both the $r_{0}$ and the rumor equilibrium point are affected by the infection rate.

\subsection{Efficiency of continuous control}

As shown in Fig. 9, if we choose $\alpha\left(k_{i}\right)=0.7, \rho\left(k_{i}\right)=$ $0.195, \gamma=0.05, \beta_{1}=0.001, \beta_{2}=0.02, p=0.01$, $d=0.01, \mu=0.005$, the rumor Spreaders 1 and Spreaders 2 exist continuously. In order to effectively suppress the spread of rumor, we use continuous control mechanism to control the rumor. Based on Theorem 6, we obtain that the rumor will disappear when we choose $\tau \geq 0.12$. Choosing $k_{1}=1$ and $k_{i} \in[1,50]$, the simulation results are shown in Figs. 10 and 11, respectively. In addition, when we choose $\alpha\left(k_{i}\right)=0.6+0.002 k$, 


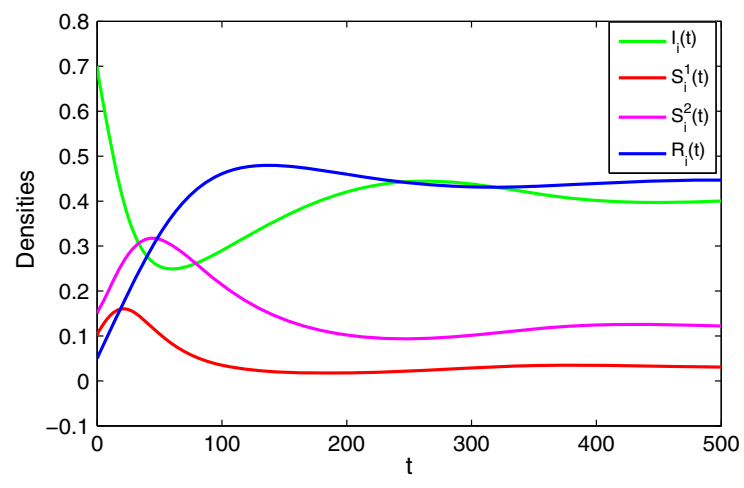

Fig. 9 Dynamics of model (1) with $r_{01}>1, r_{02}>1$ and without control

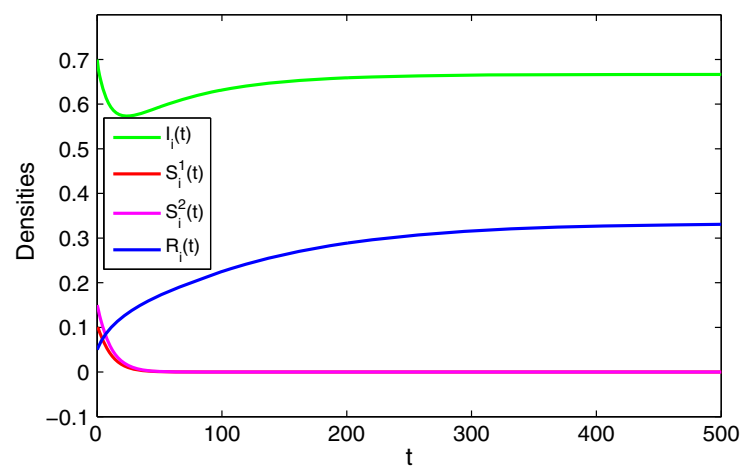

Fig. 10 Dynamics of model (33) with $\tau>0.12$ and $k_{1}=1$

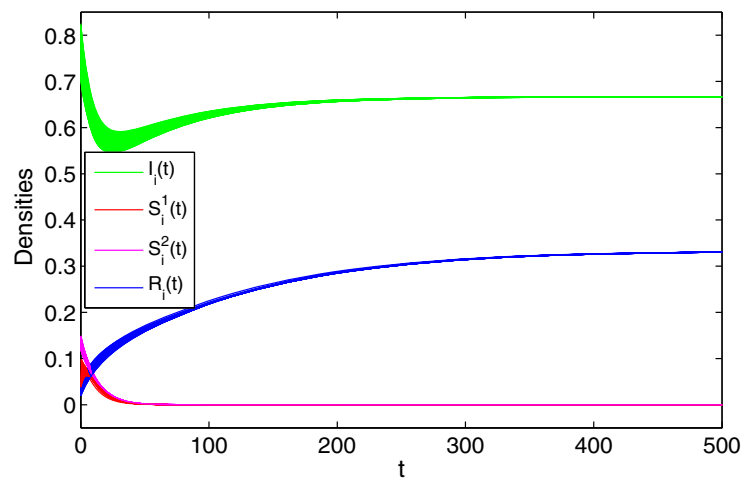

Fig. 11 Dynamics of model (33) with $\tau>0.12$ and $k_{i} \in[1,50]$

$\rho\left(k_{i}\right)=0.095+0.002 k, \gamma=0.05, \beta_{1}=0.001$, $\beta_{2}=0.02, p=0.01, d=0.01, \mu=0.005$, the state trajectories are shown in Fig. 12. All these simulation results well verify the correctness of the theories and the feasibility of the control.

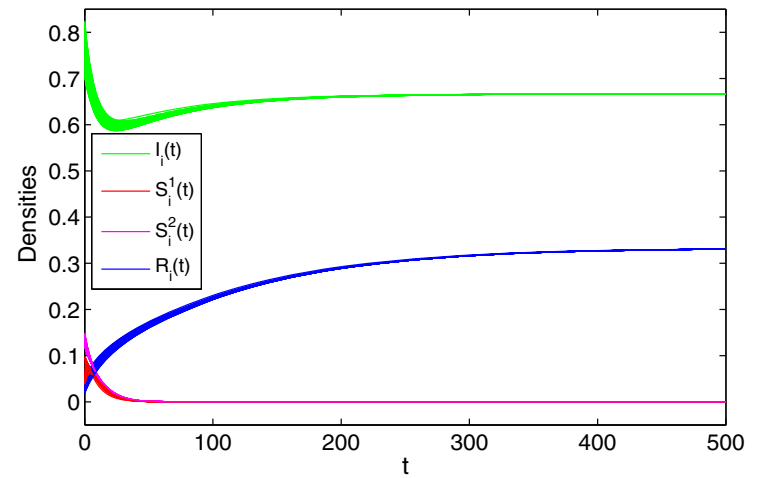

Fig. 12 Dynamics of model (33) with $\tau>0.12$ and different $\alpha\left(k_{i}\right), \beta\left(k_{i}\right)$

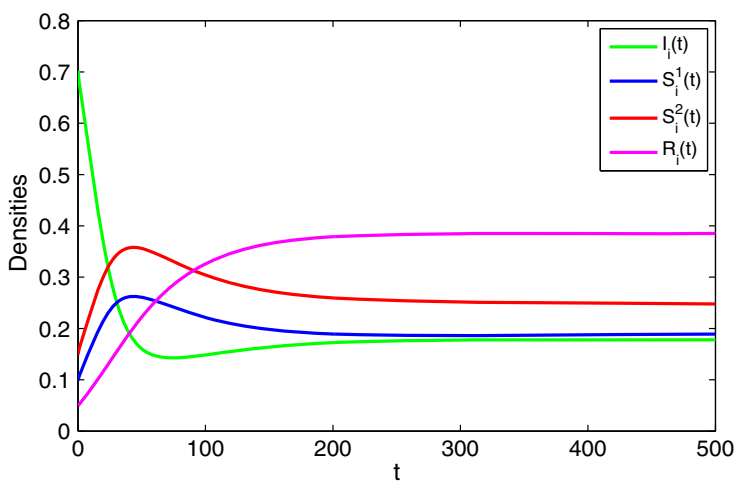

Fig. 13 Dynamics of model (1) with $r_{01}>1, r_{02}>1$ and without control

\subsection{Efficiency of event-triggered impulsive control}

For model (1), if we choose $\alpha\left(k_{i}\right)=0.5, \rho\left(k_{i}\right)=0.45$, $\gamma=0.001, \beta_{1}=0.008, \beta_{2}=0.008, p=0.01$, $d=0.01, \mu=0.002$, by simple calculation, it is derived that $r_{01}>1$ and $r_{02}>1$. As Fig. 13 shows, the rumor will persist continuously. In order to suppress rumor propagation, the event-triggered impulsive control scheme is applied. The event-trigger function is defined as $g(t)=\|e(t)\|^{2}-\frac{\varsigma}{2}\left\|X\left(t_{k}\right)\right\|^{2}-\frac{\sigma}{\left(t-t_{0}\right)^{2}}$, where $\varsigma=5, \sigma=1$. If we select $\epsilon=0.5, q=0.13$, Theorem 8 is satisfied. The state trajectories are shown in Fig. 14, in which the rumor finally dies out by using the event-triggered impulsive control. The trigger signal is shown in Fig. 15, in which 1 means triggered and 0 means not triggered. The simulation shows that the rumor will die out when we control the rumor spreaders at some discrete instants. Compared with continuous, this control scheme is more practical. 


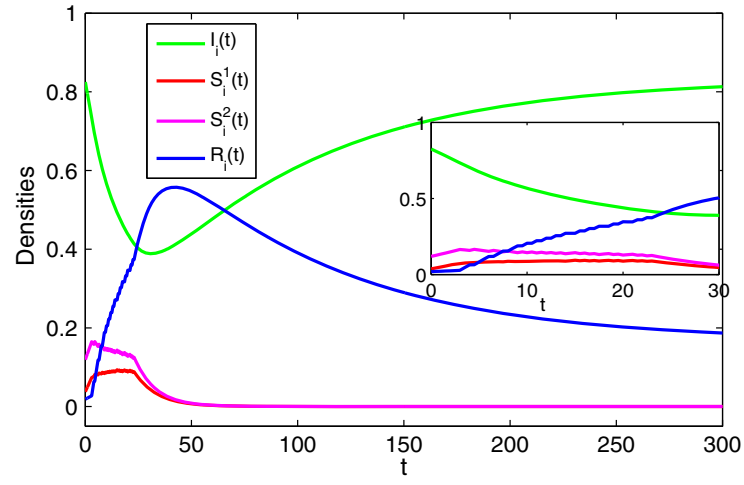

Fig. 14 Dynamics of model (1) with event-triggered impulsive control (36)

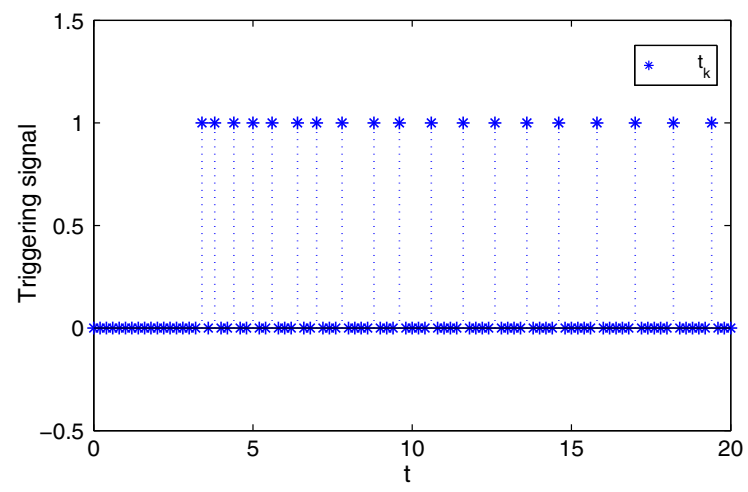

Fig. 15 The trigger signal

Remark 8 In order to analyze the correctness of the theorems, three cases for model (1) with different parameters are simulated. In each case, the simulation results verify the correctness of the theoretical analysis. When the rumor spreaders continuously exist, we propose two kinds of control schemes to suppress the spreading of the rumor. Figures 10, 11, 12, 13, 14 and 15 show that the control methods are effective. In fact, the eventtriggered impulsive control reflects that we only need to decrease the rumor spreaders at some discrete instants. This method is more resource-efficient and practical.

\section{Conclusion}

In this paper, we propose an I2SR model of rumor propagation with heterogeneous networks in a multilingual environment. In order to analyze the characteristic of rumor spreading, we calculate the basic reproduction number $r_{0}$ by using the next-generation matrix method.
The local stability and global stability of the rumor-free equilibrium point and rumor equilibrium point are studied by means of Routh-Hurwitz discriminant method and Lyapunov stability theory. In addition, two kinds of control schemes, the continuous control and eventtriggered impulsive control, are proposed to restrain the spreading of the rumor. Some related conditions are obtained to suppress the spreading of the rumor. Finally, the validity of the theoretical results is verified by numerical simulations. In practice, the communication time delay always exists. Hence, we will consider the rumor spreading model with time delays under multilingual environments in the future work.

Acknowledgements This work was supported by the National Natural Science Foundation of China (Grant No. U1703263), the Tianshan Xuesong Program (Grant No. 2018XS02), the Scientific Research Program of the Higher Education Institution of Xinjiang (Grant Nos. XJEDU2017T001, XJEDU2018Y004), the Science and Technology Project of Xinjiang Uygur Autonomous Region (Grant No. 2018Q068), the Doctoral Foundation of Xinjiang University (Grant No. 62008032), the Natural Science Foundation of Xinjiang Uygur Autonomous Region (Grant No. 2018D01C039).

\section{Compliance with ethical standards}

Conflict of interest The authors declare that they have no conflict of interest.

\section{References}

1. Daley, D., Kendall, D.: Epidemics and rumours. Nature 204(4963), 1118-1118 (1964)

2. Daley, D., Kendall, D.: Stochastic rumours. IMA J. Appl. Math. 1(1), 42-55 (1965)

3. Maki, D., Thomson, M.: Mathematical models and applications, with emphasis on social, life, and management sciences. Englewood Cliffs 10, 12 (1973)

4. Zanette, D.: Criticality behavior of propagation on smallworld networks. Phys. Rev. E 64(5), 050901 (2001)

5. Zanette, D.: Dynamics of rumor propagation on small-world networks. Phys. Rev. E 65(4), 041908 (2002)

6. Moreno, Y., Nekovee, M., Pacheco, A.: Dynamic of rumor spreading im complex networks. Phys. Rev. E 69(6), 066130 (2004)

7. Zhou, J., Liu, Z.: Epidemic spreading in complex networks. Front. Phys. China 3(3), 331-348 (2008)

8. Singh, A., Singh, Y.: Nonlinear spread of rumor and inoculation strategies in the nodes with degree dependent tie strength in complex networks. Acta Phys. Pol. B 44(1), 5 (2013)

9. Roshani, F., Naimi, Y.: Effects of degree-biased transmission rate and nonlinear infectivity on rumor spreading in complex social networks. Phys. Rev. E 85(3), 036109 (2012) 
10. Hui, W., Lin, D., Huang, Y., et al.: A variant epidemic propogation model suitable for rumor spreading in online social network. In: 2012 International Conference on Machine Learning and Cybernetics, Xian, pp. 1258-1262 (2012)

11. Liang, Z., Wang, Y.: Rumor spreading model with noise interference in complex social networks. Physica A 469, 750-760 (2017)

12. Kawachi, K., Seki, M., Yoshida, H., et al.: A rumor transmission model with various contact interactions. J. Theor. Biol. 253(1), 55-60 (2008)

13. Zhao, L., Wang, Q., Cheng, J., et al.: Rumor spreading model with consideration of forgetting mechanism: a case of online blogging LiveJournal. Physica A 390(13), 26192625 (2011)

14. Xia, L., Jiang, G., Song, B., et al.: Rumor spreading model considering hesitating mechanism in conplex social networks. Physica A 437, 295-303 (2015)

15. Zhao, L., Xie, W., Gao, H., et al.: A rumor spreading model with variable forgetting ret. Physica A 392(23), 6146-6154 (2013)

16. Schneider, B.: The reference model simpan agent-based modeling of human behavior in panic situations. In: Tenth International Conference on Computer Modeling and Simulation (uksim 2008), pp. 599-604. Cambridge, UK (2008)

17. Zhao, L., Cui, H., Qiu, X., et al.: SIR rumor spreading model in the new media age. Physica A 392(4), 995-1003 (2013)

18. Zhang, N., Huang, H., Su, B., et al.: Dynamic 8-state ICSAR rumor propagation model considering official rumor refutation. Physica A 415, 333-346 (2014)

19. Laarabi, H., Abta, A., Rachik, M., et al.: Stability analysis of a delayed rumor propagation model. Differ. Equ. Dyn. Syst. 24(4), 407-415 (2015)

20. Huo, L., Ma, C.: Dynamical analysis of rumor spreading model with impulse vaccination and time delay. Physica A 471, 653-665 (2017)

21. Wang, J., Zhao, L., Huang, R.: SIRaRu rumor spreading model in complex networks. Physica A 398, 43-55 (2014)
22. Xia, L., Jiang, G., Song, B., et al.: Rumor spreading model considering hesitating mechanism in complex social networks. Physica A 437, 295-303 (2015)

23. Liu, Q., Li, T., Sun, M.: The analysis of an SEIR rumor propagation model on heterogeneous network. Physica A 469, 372-380 (2017)

24. Huo, L., Cheng, Y., Liu, C., et al.: Dynamic analysis of rumor spreading model for considering active network nodes and nonlinear spreading rate. Physica A 506(15), 24-35 (2018)

25. He, Z., Cai, Z., Yu, J., et al.: Cost-efficient strategies for restraining rumor spreading in mobile social networks. IEEE Trans. Veh. Technol. 66(3), 2789-2800 (2016)

26. Zhu, L., Zhao, H.: Dynamical behaviours and control measures of rumour-spreading model with consideration of network topology. Int. J. Syst. Sci. 48(10), 2046-2078 (2017)

27. Wang, J., Jiang, H., Ma, T., et al.: Global dynamics of the multi-lingual SIR rumor spreading model with crosstransmitted mechanism. Chaos Solitons Fractals 126, 148157 (2019)

28. Li, J., Jiang, H., Yu, Z., et al.: Dynamical analysis of rumor spreading model in homogeneous complex networks. Appl. Math. Comput. 359, 374-385 (2019)

29. Lajmanovich, A., Yorke, J.: A deterministic model for gonorrhea in a nonhomogenous population. Math. Biosci. 28, 221-236 (1976)

30. Lasalle, J.: Stability theory for ordinary differential equations. J. Differ. Equ. 4(1), 57-65 (1968)

31. Chen, F.: On a nonlinear nonautonomous predator-prey model with diffusion and distributed delay. Comput. Appl. Math. 180(1), 33-49 (2005)

32. Gronwall, T.: Note no the derivatives with respect to a parameter of the solutions of a system of differential equations. Ann. Math. 20(4), 292-296 (1919)

Publisher's Note Springer Nature remains neutral with regard to jurisdictional claims in published maps and institutional affiliations. 